\title{
Recent highlights from GENIE v3
}

The GENIE Collaboration: Luis Alvarez-Ruso ${ }^{5}$, Costas Andreopoulos ${ }^{8,11, a}{ }^{\circ}$, Adi Ashkenazi ${ }^{9,14}$, Christopher Barry $^{8}$, Steve Dennis ${ }^{8,15}$, Steve Dytman ${ }^{10}$, Hugh Gallagher ${ }^{12}$, Alfonso Andres Garcia Soto ${ }^{4,5}$, Steven Gardiner ${ }^{3}$, Walter Giele ${ }^{3}$, Robert Hatcher ${ }^{3}$, Or Hen ${ }^{9}$, Libo Jiang ${ }^{10,16}$, Igor D. Kakorin ${ }^{7}$, Konstantin S. Kuzmin 6,7 , Anselmo Meregaglia ${ }^{2}$, Vadim A. Naumov ${ }^{7}$, Afroditi Papadopoulou ${ }^{9}$, Marco Roda ${ }^{8}$, Vladyslav Syrotenko ${ }^{12}$, Júlia Tena-Vidal ${ }^{8}$, Jeremy Wolcott ${ }^{12}$, Natalie Wright $^{9}$, Additional authors: Monireh Kabirnezhad ${ }^{13}$, and Narisoa Vololoniaina ${ }^{1}$

1 Department of Physics, University of Antananarivo, Antananarivo 101, Madagascar

2 CENBG, Université de Bordeaux, CNRS/IN2P3, 33175 Gradignan, France

3 Fermi National Accelerator Laboratory, Batavia, IL 60510, USA

4 Department of Physics, Harvard University, Cambridge, MA 02138, USA

${ }^{5}$ Instituto de Física Corpuscular (IFIC), Consejo Superior de Investigaciones Científicas (CSIC) y Universitat de València (UV), 46980 Paterna, València, Spain

6 Alikhanov Institute for Theoretical and Experimental Physics (ITEP) of NRC "Kurchatov Institute", Moscow 117218, Russia

7 Joint Institute for Nuclear Research (JINR), Dubna, Moscow region 141980, Russia

8 Department of Physics, University of Liverpool, Liverpool L69 7ZE, UK

9 Department of Physics, Massachusetts Institute of Technology, Cambridge, MA 02139, USA

10 Department of Physics and Astronomy, University of Pittsburgh, Pittsburgh, PA 15260, USA

11 Particle Physics Department, U.K. Research and Innovation, Science and Technology Facilities Council, Rutherford Appleton Laboratory, Oxfordshire OX11 0QX, UK

12 Department of Physics and Astronomy, Tufts University, Medford, MA 02155, USA

13 Department of Physics and Astronomy, York University, Toronto, ON M3J 1P3, Canada

14 Present address: Tel Aviv University, Tel Aviv, Israel

15 Present address: University of Cambridge, Cambridge, UK

16 Present address: Virginia Polytechnic Institute and State University, Blacksburg, VA, USA

Received 22 June 2021 / Accepted 23 September 2021 / Published online 8 December 2021 (C) The Author(s) 2021

\begin{abstract}
The release of GENIE v3.0.0 was a major milestone in the long history of the GENIE project, delivering several alternative comprehensive neutrino interaction models, improved charged-lepton scattering simulations, a range of beyond the Standard Model simulation capabilities, improved experimental interfaces, expanded core framework capabilities, and advanced new frameworks for the global analysis of neutrino scattering data and tuning of neutrino interaction models. Steady progress continued following the release of GENIE v3.0.0. New tools and a large number of new physics models, comprehensive model configurations, and tunes have been made publicly available and planned for release in v3.2.0. This article highlights some of the most recent technical and physics developments in the GENIE v3 series.
\end{abstract}

\section{Introduction}

The release of GENIE v3.0.0 was a major milestone in the long history of the GENIE project [1]. The associated technical and physics modelling developments underlined the dual role of GENIE in (a) maintaining the single universal platform for delivering well-validated and state-of-the-art physics simulations directly into the established Monte Carlo (MC) simulation chains of nearly all neutrino experiments, and (b) taking the leading role in the development, validation, characterisation and tuning of comprehensive neutrino

\footnotetext{
a e-mail: publications@genie-mc.org (corresponding author)
}

simulations, that incorporate descriptions for all relevant processes across the full kinematic space accessible by different types of neutrino experiments. Addressing the community demand for alternative models, GENIE v3.0.0 amalgamated large collections of modelling elements, many of which were developed with strong community support, into a number of distinct and relatively consistent comprehensive model configurations that were validated, characterised, tuned and deployed as a whole [2]. They were the seeds around which many modelling developments have coalesced, leading to more well-motivated variants and tunes. The maturation of the collaborative GENIE development paradigm, along with the substantial effort invested in curating extensive data archives of neutrino, electron and hadron 
scattering data, developing advanced frameworks for data/MC comparisons, tuning, and continuous integration underpinned a marked improvement both in the volume of deployed simulations and in release frequency.

Steady progress continued following the release of GENIE v3.0.0, with a large number of new modelling elements, comprehensive model configurations and tunes planned for release in v3.2.0. A comprehensive description of GENIE v3 is much beyond the scope of this article, which will only highlight some of the most recent technical and physics developments, with particular emphasis on new developments that followed the release of v3.0.0. ${ }^{1}$

This article is organised as follows: technical developments, with particular emphasis on the core generator framework improvements, a new event library interface that allows experiments to re-use the mature GENIE experimental interfaces with third-party neutrino generators, and the GENIE global analysis of neutrino scattering data are discussed in Sect. 2. New developments in the description of neutrino, electron and hadronnucleus scattering, which are discussed in Sect.3, are a focal point of this article. Special emphasis is given in the expanded modelling of the nuclear ground state, the careful validation and improvement of electron-nucleus scattering simulations, new models of zero-pion production (i.e. quasielastic (QE) and multi-nucleon mechanisms such as meson exchange current (MEC) or twoparticle-two-hole $(2 p 2 h)$ excitations in general), singlepion production (including both resonant (RES) and non-resonant (NONRES) contributions to the amplitude), new models for coherent $(\mathrm{COH})$ single-photon production and coherent elastic scattering, and new advanced models of final state interactions (FSI) delivered through interfaces to the INCL ${ }^{2}[3]$ and Geant4 [4] codes, a complete new set of high-energy simulation modules including a next-to-leading order (NLO) deepinelastic scattering (DIS) simulation, and a high-level description of all recent comprehensive configurations and tunes. Finally, in Sect. 4, we highlight recent developments in GENIE beyond the Standard Model (BSM) modelling capabilities, which form an important component of the overall program of work in GENIE, in support of the full science program of modern neutrino experiments.

\section{Technical updates}

One of the most visible updates is the evolution of GENIE into a suite of separate products, maintained in different repositories. Notable GENIE opensource products include the: (a) Generator, containing all GENIE physics modules, experimental inter-

\footnotetext{
1 A complete description of GENIE v3 will appear in future publications or in the manual available on the GENIE website http://www.genie-mc.org/.

2 Liège Intranuclear Cascade.
}

faces (flux and detector geometry drivers) and a host of generic and specialised event generation applications, (b) Reweight, containing procedures for propagating generator uncertainties, (c) Lamp, which includes a collection of scripts for building GENIE and necessary external packages, (d) UnitTests, and (e) AVS-CI, containing GENIE's Automated Validation Suite for Continuous Integration. Codes for data/MC comparisons that in earlier GENIE v2 revisions existed within the generator, were extracted and formed the basis of additional products that were the focus of substantial development from the core GENIE team over the past few years. They include the: (a) Comparisons, containing curated archives of neutrino, charged-lepton and hadron scattering data, as well as highly-developed software to produce a comprehensive set of data/MC comparisons, (b) Prof-GENIE, implementing the GENIE interface to the Professor tool [5], and (c) Tuning, containing the procedures implementing the GENIE global analysis of neutrino scattering data. The latter group of products plays a central role in the development and characterization of GENIE comprehensive models and tunes and in the GENIE global analysis. While this analysis is in active development, these products do not have open source releases and, therefore, a detailed description of the numerous developments therein is not in the scope of this brief article. All the repositories corresponding to the suite of GENIE products changed from SVN [6] to Git [7], and they are hosted in the GENIE organization on GitHub, https://github.com/ GENIE-MC.

At a more detailed level, some of the most visible technical updates were implemented with the goal of supporting an expanded physics content and they range from framework improvements to an interface for a systematic tuning procedure. Some highlights are listed below.

\subsection{Core software framework improvements}

From the user point of view, the changes in the framework are all related to configuration and mostly geared toward internal consistency. As the different code modules are largely independent, it was possible to configure physics parameters to be different for each model leading to the generation of events obtained with inconsistent physics settings. For example different models could use different values of the coupling constants. To solve this issue, the concept of common parameter was introduced: they are parameters sets that are configurable by the user and yet uniquely defined in memory allocations accessible from every algorithm.

As it was for version 2, the event generation is subdivided in different processes. In this context, processes are labelled after different scattering types (QE, RES, DIS, etc.) but they are GENIE terminology to identify different event generation algorithms that are tailored for specific final states. In general, processes are not universal and their definitions are only valid within the GENIE software. This modular structure 
has a large degree of configuration: for each process, the system offers a number of alternative models to be used for event generation, see Sect.3. In previous GENIE releases, only one model-process mapping was suggested by the out-of-the-box configuration, despite the availability of alternative models. Yet, there was no guidance on how to correctly use different configurations according to author and developers. Hence, it was easy to come up with inconsistencies between the model configuration for different processes that were supposed to be used together to get a correct comprehensive physics simulation. An example of inconsistent configuration is using the Valencia model (see Sect. 3.3) with a relativistic Fermi gas nuclear model. This issue was addressed in GENIE v3 by introducing the concept of comprehensive model configuration (CMC) that is a consistent process-model association. Considering that GENIE already has about 20 different processes for neutrinos alone, CMC definitions are quite complex objects and they need to be effectively named so that the community can use them unambiguously. For this purpose, the collaboration developed a specific naming convention that is described in the manual. Section 3.9 will summarise the names and the physics relevant for for this paper. These CMCs are aimed at specific types of experiment and can be expanded according the needs of collaborations.

A final element to be mentioned is related to the internal PDG [8] library. The PDG values used so far in GENIE have not been changed to keep the predictions consistent with past versions. Now users will have the possibility to use different PDG library configurations that are tune dependent.

\subsection{The new GENIE tuning process}

Tuning is a necessary step for all MC generators. In the specific case of neutrino generators, it is required to merge together different models to avoid double counting, since there is no single model able to cover all possible interactions across the whole energy range. Indeed, development of a global analysis of scattering data for the tuning and uncertainty characterization of comprehensive neutrino interaction models has been a central activity of the core GENIE team over the past few years. The GENIE Generator is the main outlet for the GENIE global analysis results, and our goal is that, for each supported comprehensive model, several selected tuned versions shall be made available.

The GENIE global analysis was made possible through the continued development of curated data archives, and their successful interface to the Professor tool [5]. This interface enabled the efficient implementation of complex multi-parameter brute-force scans and removed substantial global analysis limitations by decoupling it from event reweighting procedures that, for all but the most trivial aspects of our physics domain, require substantial development time and are not exact, or even possible at all. Specifically, Professor 'reduces the exponentially expensive process of brute- force tuning to a scaling closer to a power law in the number of parameters, while allowing for massive parallelisation' [9].

This concept goes beyond the existing reweighting scheme since it allows the tuning of parameters that are not normally reweightable. We expect to be able to develop a reweighting tool based on this method for GENIE v4. After a specific experimental flux is defined as an input, the phase space of each interaction can be decomposed in bins seen as an observable. Eventually we expect the users and experiments to build their own response functions to allow the reweighting of their predictions according to the statistical output of the tunes based on this technology.

\subsection{Event library}

The neutrino flux and geometry interfaces and the wealth of mature and well-tested drivers implementing these interfaces constitute one of the most well known and desirable GENIE features that has catalysed GENIE adoption and enabled seamless integration in the full MC simulation chain of all current and nearfuture neutrino experiments. No other physics generator provides an equivalent and equally comprehensive and mature toolkit and experimental interfaces. There is a strong community desire to reuse the GENIE experimental interfaces to test alternative physics generators. This drove the implementation of an "Event Library" interface [10] and the development of a generic EvtLib GENIE generator.

The purpose of the EvtLib generator is to read from an external library of cross sections and pre-computed final particle kinematics (most likely computed using an alternative neutrino generator). For each interacted neutrino selected by GENIE, the generator will use the appropriate cross section from the file, and then use the kinematics from the library entry with the closestmatching energy. Within the limits of the library statistics, this will then reproduce the physics of the external generator, but making use of the flux and geometry handling of GENIE. The details of the event library file structure are described in the code and in the manual [11].

\section{Interaction modeling improvements}

Neutrino-nucleus interactions are very important to many experiments and this remains a central area of effort within the GENIE collaboration. Chargedcurrent-neutrino interactions without final-state mesons ( $\mathrm{CC} 0 \pi$ interactions) will dominate the expected signal in future precision oscillation measurements by the Short-Baseline Neutrino program [12] and HyperKamiokande [13]. Significant GENIE development effort has recently been devoted to the implementation of new models of quasielastic and $2 p 2 h$ interactions (see Sect.3.3). Many channels will be important for the upcoming Deep Underground Neutrino Experiment 
(DUNE) [14], especially because the higher average beam energy will enhance the role of more inelastic event topologies. Resonance production and FSI will be very important for DUNE. Recent improvements in those areas are discussed in Sects. 3.4 and 3.6, respectively.

At the same time, significant efforts have gone into new capabilities at very low energies (Sect. 3.8) and very high energies (Sect.3.7). In addition, the importance of electron scattering Sect. 3.2 to determine nuclear structure Sect. 3.1 and vector interactions is expanding. These three directions greatly enhance the reach of GENIE into new experiments.

\subsection{Nuclear ground state}

At energies relevant for accelerator neutrino experiments, a variety of nucleon-level hard scattering processes (principal interactions), such as resonance production, must be considered when preparing a comprehensive lepton-nucleus interaction model for use in an event generator. However, two aspects of such a comprehensive model will be common to all interaction modes: a description of the nuclear ground state (the subject of this section) and a treatment of intranuclear rescattering due to hadronic final-state interactions (the subject of Sect. 3.6).

There are a variety of methods to model the nuclear ground state. Improving these models is an ongoing process within GENIE. At present, the nuclear ground state is represented by a spectral function $P(\mathbf{p}, E)$ which describes the probability that a nucleon involved in a lepton-nucleus interaction will have an initial 3momentum $\mathbf{p}$ and removal energy $E$. In the historical default model used since GENIE v2, the initial nucleon momentum is sampled according to the relativistic Fermi gas (RFG) treatment of Bodek and Ritchie [15]. This version of the RFG has non-interacting nucleons up to the Fermi momentum $k_{F}$, which is determined from inclusive electron scattering. It also accounts for short-range nucleon-nucleon correlations [16] by adding a high-momentum tail above $k_{F}$ to the usual distribution. A fixed, isotope-specific removal energy $E$ is used in all cases. Pauli blocking in quasielastic interactions is implemented by requiring the final-state nucleon momentum to exceed $k_{F}$. The Bodek-Ritchie RFG continues to be used in multiple GENIE CMCs mainly for higher energy processes and connection with past modeling.

Two newer nuclear model implementations are available for all target nuclides in GENIE v3.2. The first of these is a local Fermi gas (LFG) model based on the work of the Valencia group [17] and various other publications. Under this approach, the high-momentum tail is neglected, and $k_{F}$ is a function of radius obtained from the nucleon number density $\rho(r)$ via

$$
k_{F}(r)=\left(3 \pi^{2} \rho(r) / 2\right)^{1 / 3}
$$

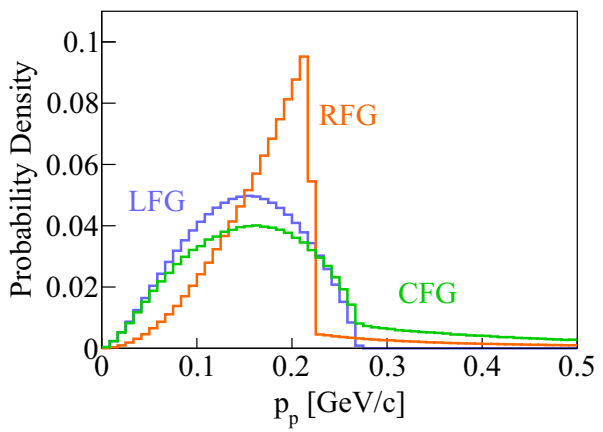

Fig. 1 Initial nucleon momentum magnitude distributions according to the GENIE implementation of relativistic Fermi gas, local Fermi gas and correlated Fermi gas models

The implementation of this model underpins a related treatment of quasielastic and two-particle-two-hole interactions (see Sect. 3.3), in which nuclear effects such as long-range correlations and Coulomb corrections are handled according to the same local density approximation in the Valencia model.

A variation of the original LFG model, called the correlated Fermi gas (CFG) [18], has also been added in GENIE v3.2. The CFG keeps the radial dependence of the LFG model while adding a high-momentum tail that lies above the local $k_{F}$. The original LFG distribution is renormalized to ensure that a given fraction of initial-state nucleons is found in the tail. The current default of $20 \%$ is based on electron scattering measurements [18] and may be adjusted in future GENIE tuning efforts. Both the LFG and CFG implementations in GENIE use a fixed nucleon removal energy which is identical to that used by the Bodek-Ritchie RFG. Figure 1 shows the $|\mathbf{p}|$ distribution predicted by each of the three models of the nuclear ground state discussed above, where $\mathbf{p}$ is the initial nucleon momentum.

\subsection{Electron-nucleus scattering}

Since neutrinos and electrons are both leptons, they interact with atomic nuclei in similar ways. Electrons interact via a vector current and neutrinos interact via vector and axial-vector currents. Electron-nucleus scattering data allow for more precise measurements than $\nu$-nucleus scattering due to an interaction rate that is $O\left(10^{7}\right)$ times higher, thanks to the stronger electromagnetic interaction over the weak neutrino coupling. Furthermore, the knowledge of the incoming flux is more constrained. Specifically, monochromatic beams allow for proper kinematic reconstruction, reducing the systematic uncertainty. These higher quality data allow for powerful model constraints on the vector part of leptonnucleus cross sections. Key nuclear effects common to electron and neutrino probes, such as the nuclear ground state and hadronic FSIs, can also be studied in detail.

From GENIE version 3.00.06, significant improvements were made for aspects of both neutrino and 
electron cross section modeling [19]. Significant errors were corrected, including a mistake in the mathematical expression used for the Rosenbluth QE differential cross section [20], a missing Lorentz boost in the $2 p 2 h$ interaction affecting both electrons and neutrinos, and incorrect electron couplings used in the RES interactions. As a result, all electron scattering channels were effected and agreement with data improved. In general, wherever possible, the electron treatment was updated to be significantly more similar to the neutrino one and to use the same computer code.

The GENIE collaboration is in the process of benchmarking the electron scattering predictions against existing inclusive electron scattering data for different target nuclei, beam energies and scattering angles [19], as can be seen in Fig. 2. The physics content of the model configurations shown is described in Sect. 3.9. ${ }^{3}$ The agreement is very good for the kinematic region dominated by QE processes due to the bug fixes and adoption of newer models. On the other hand, the simulation is well above the data in the resonance region. This is largely due to deficiencies in modelling of the fundamental scattering process rather than the treatment of nuclear effects. In particular, the existing GENIE tunes to measurements with hydrogen and deuterium targets [2] only use neutrino (as opposed to electron) data. As a partial accounting, we have added the Bosted-Christy model $[21,22]$ as an alternate cross section for use in user-driven reweighting. Similar electronrelated activities are taking place in other generators like GiBUU [23], NEUT [24] and NuWro [25].

The community is also in the process of reviewing and improving the electron scattering data. For example, high-statistics datasets from the CLAS6 detector at Jefferson Laboratory were analysed [27] and new experiments ( $e 4 \nu$, Mainz, and LDMX) designed to support neutrino interaction experiments will start data taking in 2021 [28-30]. Major emphasis in these new measurements will be placed on a detailed description of the hadronic part of the final state. These new data will be necessary to benchmark the performance of cross sections and nuclear models used by generators.

\section{3 $\mathrm{CC} 0 \pi$ cross sections}

Initial efforts to isolate CCQE interactions in neutrinonucleus scattering data proved to have significant model dependence. Hence, experimental attention is now focused on $\mathrm{CC} 0 \pi$ event topologies which involve three underlying processes within GENIE. CCQE and $2 p 2 h$ are the main contributors; nonetheless, pion production followed by intranuclear absorption also contributes significantly to this channel.

The GENIE v2 historical default model for chargedcurrent quasielastic scattering was based on the Llewellyn Smith [31] formalism: the expression for the

3 Please note that the GENIE configuration referred to in Ref. [19] as GSuSAv2 or GTEST19_10b_00_000 is now labeled as GEM21_11b_00_000 in GENIE version 3.02.00. The previous "test" configuration was promoted to a full CMC.

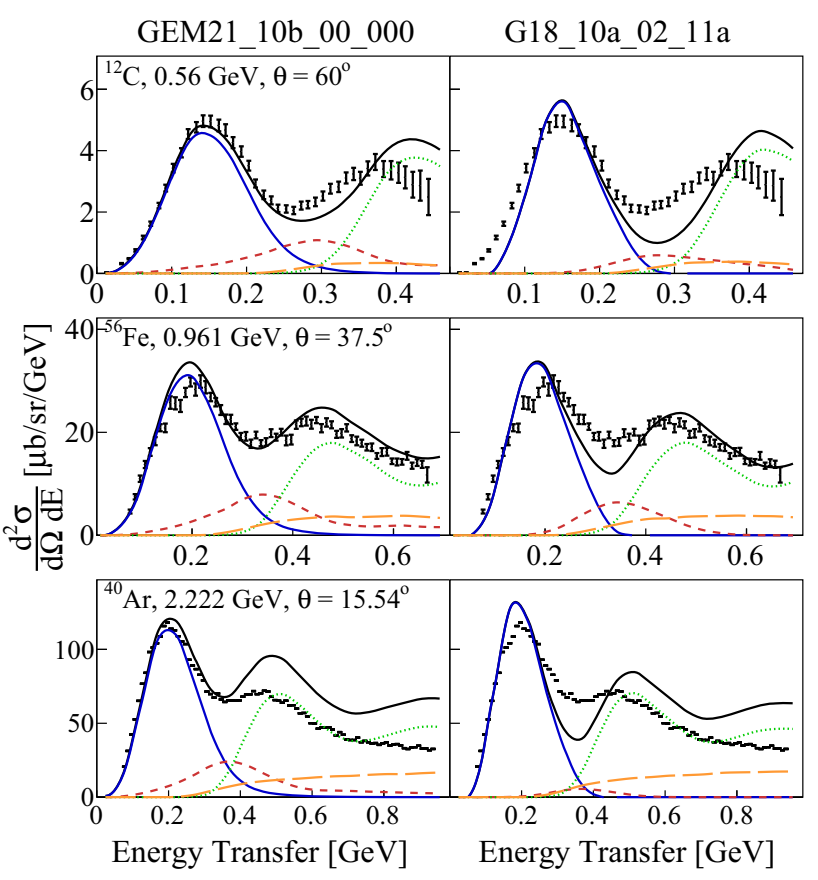

Fig. 2 Comparison of inclusive $\left(e, e^{\prime}\right)$ scattering cross sections for data and for GENIE [19]. (left) data vs GEM21_10b_00_000 and (right) data vs G18_10a_02_11a. (top) carbon at $E_{0}=0.56 \mathrm{GeV}, \theta_{e}=60^{\circ}$ and $Q_{\mathrm{QE}}^{2} \approx 0.24$ $\mathrm{GeV}^{2}$, (middle) iron at $E_{0}=1 \mathrm{GeV}, \theta_{e}=37.5^{\circ}$ and $Q_{\mathrm{QE}}^{2} \approx 0.32 \mathrm{GeV}^{2}$, and (bottom) argon at $E_{0}=2.22 \mathrm{GeV}$, $\theta_{e}=15.5^{\circ}$ and $Q_{\mathrm{QE}}^{2} \approx 0.33 \mathrm{GeV}^{2}$. Black points show the data, solid black lines show the total GENIE prediction, colored lines show the contribution of the different reaction mechanisms: (blue) QE, (red) MEC, (green) RES and (orange) DIS. Due to the fixes mentioned in Sect.3.2, this figure differs significantly from those produced with previous versions of the code, for example [26, Fig. 2]

hadronic part of the cross section is taken to be the same as for a free nucleon. The corresponding cross section for scattering on a complex nucleus is then computed by correcting for Pauli blocking and binding energy, averaging over the initial nucleon momentum distribution (see Sect.3.1), and then multiplying by the total number of neutrons (protons) for an incident neutrino (antineutrino).

The Llewellyn Smith approach is still available in GENIE v3.2 and remains a good model for neutrino energies above roughly $2 \mathrm{GeV}$. Two additional CCQE models which contain details that are important for lower energy neutrinos have now also been implemented. One of these is based on the formalism of the Valencia group [17] and makes two major refinements beyond Llewellyn Smith. First, long-range nucleon correlations are treated in a Random Phase Approximation (RPA) approach. Corrections for these are included as density-dependent modifications to the free-nucleon hadronic tensor. Second, corrections for the final-state Coulomb interaction of the outgoing charged lepton are introduced using a strategy similar to the "modified effective momentum approximation" proposed by Engel 
[32]. The radial dependence of both of these nuclear effects is taken into account by relying on the local Fermi gas model described in Sect. 3.1.

In the GENIE implementation of the Valencia model, a correction for binding energy is made by assigning an off-shell total energy to the initial struck nucleon. The QE cross section is then calculated under the de Forest prescription [33]: an effective energy transfer is used which is reduced by the amount of energy needed to put the initial nucleon on the mass shell. Nieves et al. also recommend using an effective energy transfer in the original Valencia model publication, but their approach is different. Rather than considering nucleon knock-out, the authors adjust the energy transfer $q^{0}$ via ${ }^{4}$

$$
q^{0} \rightarrow q^{0}+\Delta M-\Delta E_{F},
$$

where $\Delta E_{F}=E_{F}^{N_{i}}-E_{F}^{N_{f}}$ is the difference in Fermi energies

$$
E_{F}^{N}=\sqrt{m^{2}+k_{F}^{2}(r, N)} \quad N \in n, p
$$

between the initial $\left(N_{i}\right)$ and final $\left(N_{f}\right)$ nucleon species and $m$ is the nucleon mass. The parameter $\Delta M=M_{i}-$ $M_{f}$ is the difference in ground-state masses between the initial nucleus $\left(M_{i}\right)$ and a final nucleus $\left(M_{f}\right)$ that includes the outgoing nucleon.

While either binding energy correction would be a defensible choice for simulations of CCQE events, the default GENIE approach was preferred because it provides a consistent treatment that allows electron scattering data to be used to determine a specific value for the nucleon binding energy. In contrast, the procedure given in Eq. 2 leads to no correction at all for electromagnetic scattering ( since $\Delta M=\Delta E_{F}=0$ ). Constraints from electron scattering data on the value of the nucleon binding energy to use in neutrino event generators were recently examined in detail in Ref. [34].

The left-hand plot in Fig. 3 shows a comparison of GENIE calculations to MiniBooNE $\mathrm{CC} 0 \pi$ data [35] in which both the default binding energy treatment (solid green) and original Valencia model approach (dotted violet) have been applied: the two predictions yield reasonably similar results. Interestingly, better agreement with the data is seen if binding energy corrections are neglected entirely (dashed blue). However, a naïve attempt to match the MiniBooNE result by ignoring binding energy cannot be justified in light of GENIE comparisons to electron scattering data, such as the one shown in the right-hand plot of Fig. 3. The location of the quasielastic peak shown in this plot cannot be successfully reproduced without including a correction for binding energy. The tails and the width of the distribution are sensitive to other aspects of the model which are not relevant for this discussion.

A second new CCQE model in GENIE [37] implements the SuSAv2 treatment [38]. Under this approach,

\footnotetext{
4 Eq. 43 in Ref. [17] is equivalent to Eq. 2 but uses different notation.
}

the nuclear responses are calculated using scaling functions based on Relativistic Mean Field (RMF) theory. A precomputed table of these responses, defined on a twodimensional grid in energy and momentum transfer, is interpolated for efficient sampling of final-state lepton kinematics. Handling of such tabular input for QE and $2 p 2 h$ models is an important new capability added in v3.2. A factorisation strategy is employed to simulate the outgoing nucleon: the leptonic 4-momentum transfer is applied to a nucleon drawn at random from the initial-state single nucleon distribution (see Sect. 3.1). The limitations of this approximation are considered in Ref. [37].

New models of $2 p 2 h$ interactions have also been recently implemented in GENIE following the Valencia $[39,40]$ and SuSAv2 [41,42] approaches. These provide theory-driven alternatives to the Empirical model [26] available since late releases of GENIE v2. In contrast to the QE case, the SuSAv2-MEC model is based on a relativistic Fermi gas description of the nucleus. Both new $2 p 2 h$ models rely on an implementation strategy similar to the one used for SuSAv2 QE: inclusive differential cross sections are calculated using tables of nuclear responses [43], and the sampled 4-momentum transfer is then imparted to a cluster of two nucleons chosen from the single-nucleon ground-state nuclear model. Separate nuclear response tables are provided based on the isospin composition of the struck nucleon pair ( $n n, p n$, or $p p)$, which is chosen by comparing the relative contributions to the inclusive differential cross section at fixed lepton kinematics. The combined Valencia $\mathrm{QE}+2 p 2 h$ model is available in GENIE only for CC neutrino scattering, while SuSAv2 may be applied to electron scattering as well.

The left (right) plot in Fig. 4 illustrates some representative differences between the three GENIE CCQE $(2 p 2 h)$ models described above. The RPA corrections included in the Valencia CCQE model lead to a suppression of low- $Q^{2}$ events, shown here for $\nu_{\mu}$ scattering on argon in MicroBooNE. All three $2 p 2 h$ models predict distinct distributions of the hadronic invariant mass $W$, with the Valencia calculation uniquely splitting the strength into two peaks. Neutrino detectors capable of measuring pairs of final-state nucleons, such as liquid argon time projection chambers [44], may provide helpful constraints on these $2 p 2 h$ model differences in the future.

\subsection{Pion production}

Treatment of pion production in GENIE is of great interest because many aspects of the underlying theory are complicated and poorly understood. The existing GENIE models are all based on the phenomenological approach of Rein, and Sehgal (RS model) [45,46], that aimed at describing pion production in the resonance region using nucleon-to-resonance transition matrix elements calculated with the relativistic quark model of Feynman, Kislinger, and Ravndal. The original RS model (without the interference between resonances) 


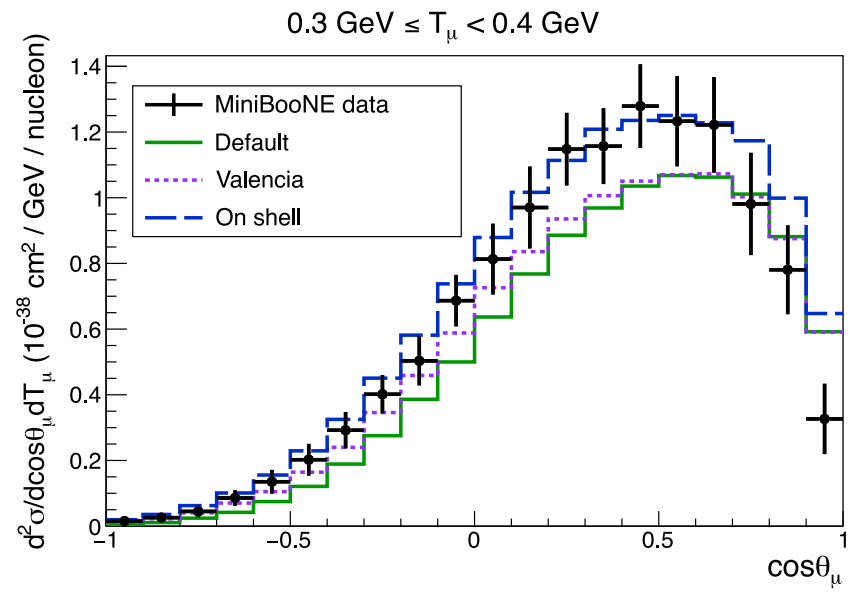

Fig. 3 Impact of nucleon binding energy effects on GENIE cross section predictions. LEFT: MiniBooNE double-differential $\mathrm{CC} 0 \pi$ cross section compared to GENIE G18_10a_02_11b predictions calculated with variations to the binding energy correction. RIGHT: Differential cross section predictions for inclusive $560 \mathrm{MeV}$ electron scattering on ${ }^{12} \mathrm{C}$ at $\theta=60^{\circ}$. The solid green histogram shows the nominal prediction using the G18_10a_02_11b CMC. The

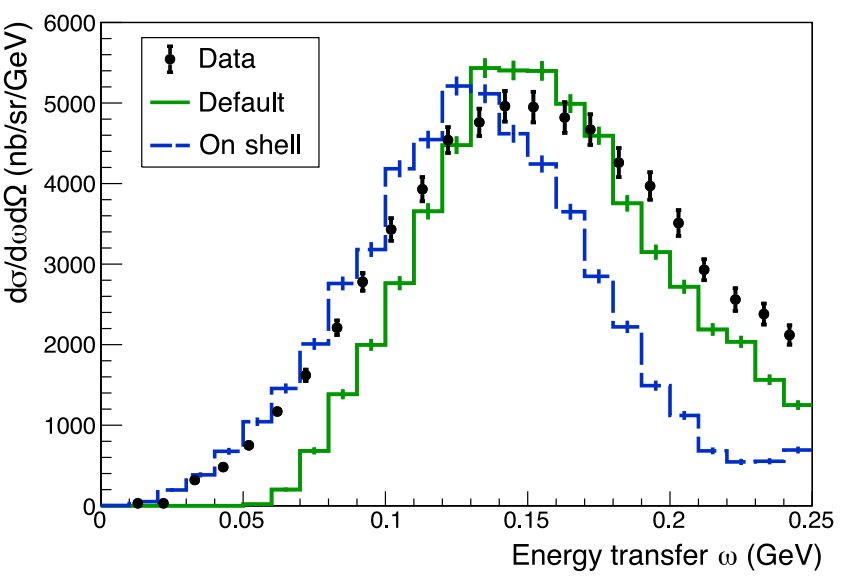

dashed blue histogram shows the same cross section when no correction for binding energy is applied. Data points are taken from Ref. [36]. The $Q^{2}$ values represented by the data points in the right-hand plot (ranging between 0.17 and $0.31 \mathrm{e} \mathrm{GeV}^{2}$ ) are roughly comparable to the MiniBooNE flux-averaged mean $Q^{2} \approx 0.4 \mathrm{GeV}^{2}$ predicted by the GENIE G18_10a_02_11b simulation
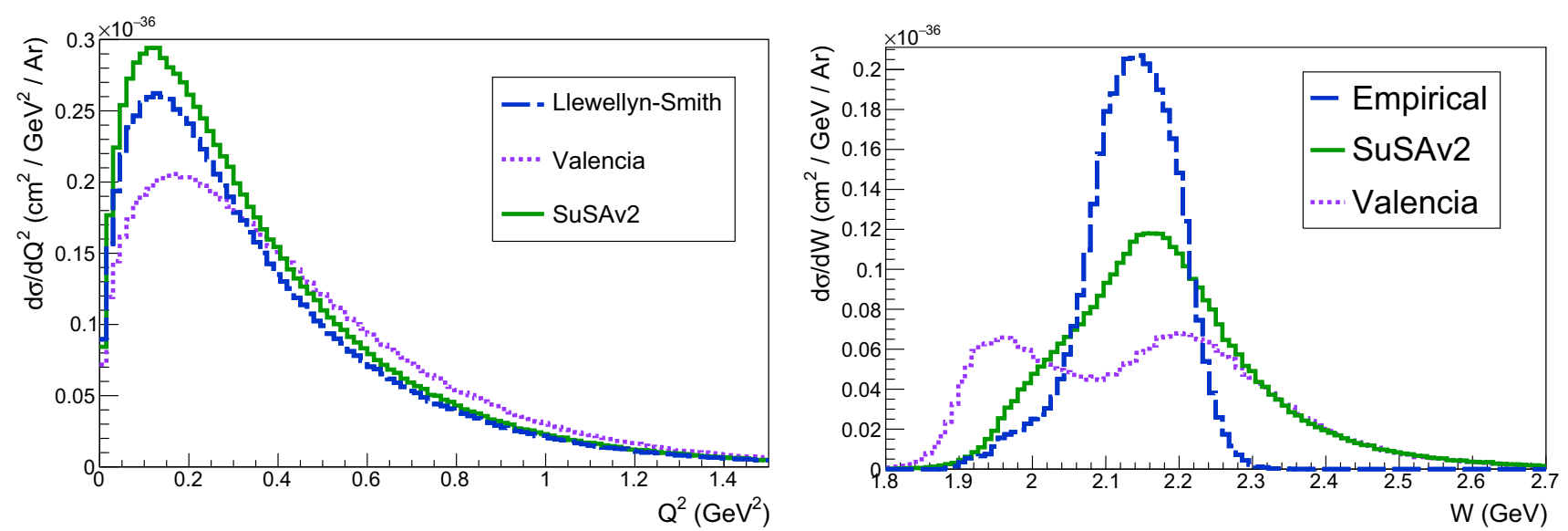

Fig. 4 Differential cross sections predicted by several different GENIE treatments of $\nu_{\mu}$ charged-current quasielastic (left) and two-particle-two-hole (right) interactions on argon. All distributions shown are averaged over the MicroBooNE $\nu_{\mu}$ flux

has always been in GENIE. The non-resonant background (NRB) in GENIE is simulated by DIS contribution [47] with the structure functions proposed by Bodek and Yang $[48,49]$. This model extends down to $\pi \mathrm{N}$ threshold; its normalization is adjusted in the "resonance-dominated" region so that the summed response in this region agrees with $\nu \mathrm{H} / \mathrm{D}$ inclusive cross section data. In v2.10, improvements by by Kuzmin, Lyubushkin, and Naumov (KLN) $[50,51]$ and by Berger and Sehgal (BS) [52] were introduced to account for nonzero lepton mass, lepton polarization, and pion pole contributions. At the same time, updated form factors for pion production were proposed by Graczyk and Sobczyk [53] and were added to GENIE [54]. The RS model parametrises the axial transition form factors in terms of a common parameter, the axial-vector mass, $M_{A}^{\mathrm{RES}}$, which is adjusted in each physical tune, and the default value is $1.12 \mathrm{GeV}$ [55]. Details on the previous implementations are given in Refs. [11,56], and the approach to combining resonant and non-resonant contributions taken in GENIE 3.0 is described in Ref. [2].

In v3, normalization of the Breit-Wigner distributions (code has a probability distribution based on a function with a very long tail) was treated more carefully. Various problems with the helicity amplitudes (e.g. sign errors) were fixed. The result was small changes in the leading $\Delta(1232)$ amplitude and $\sim 15 \%$ changes in small amplitudes such as $P_{13}(1720)$ and for $\bar{\nu}$.

An upcoming GENIE release will include an implementation of the new single-pion $(1 \pi)$ production model [57-60] (the MK model) based on the Rein's formalism [61] and the KLN/BS lepton-mass treatment of 


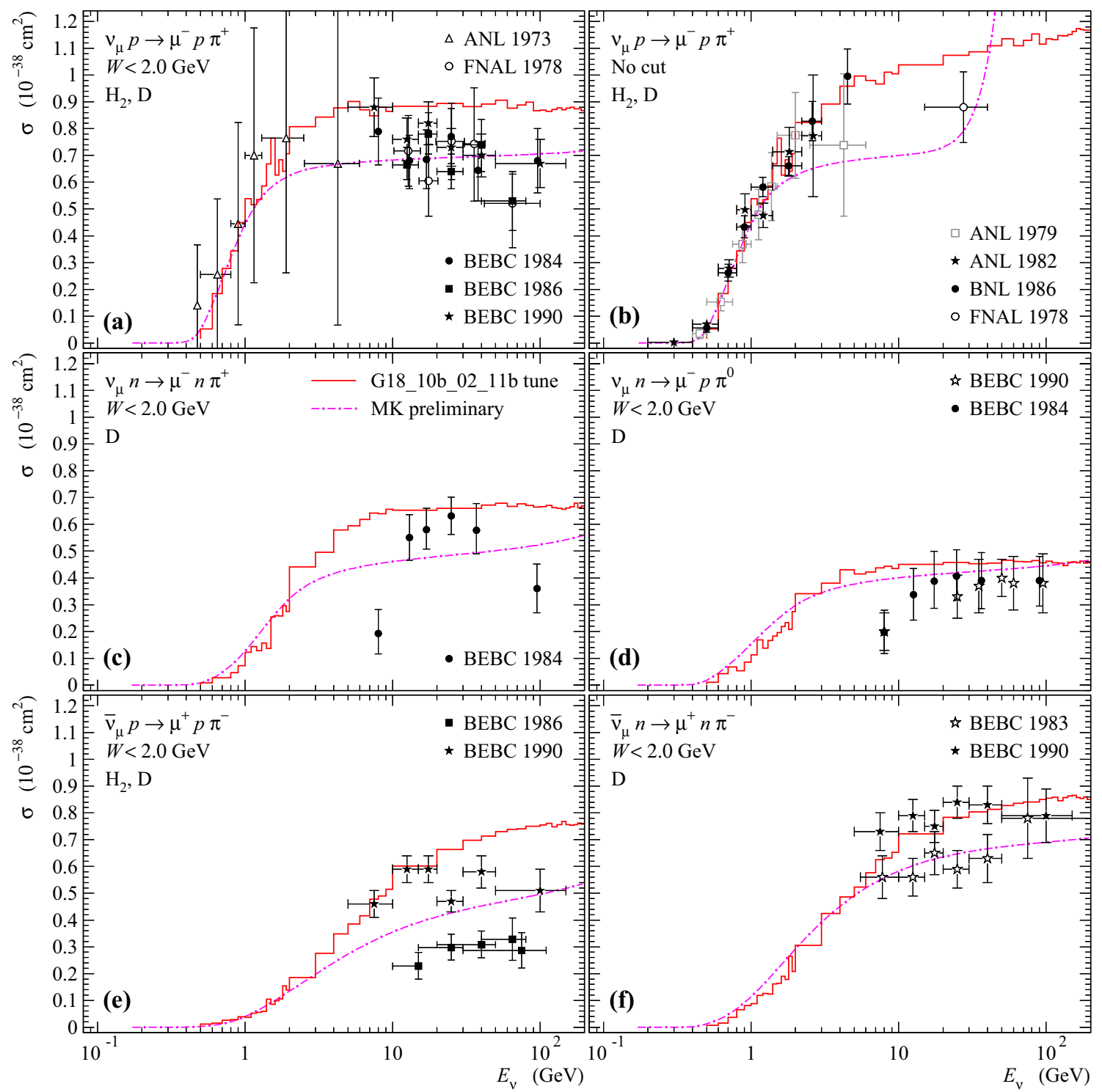

Fig. 5 Total cross sections of the reactions $\nu_{\mu} p \rightarrow \mu^{-} p \pi^{+}$ (a), (b), $\nu_{\mu} n \rightarrow \mu^{-} n \pi^{+}$(c), $\nu_{\mu} n \rightarrow \mu^{-} n \pi^{+}$(c), $\nu_{\mu} n \rightarrow$ $\mu^{-} p \pi^{0}(\mathrm{~d}), \bar{\nu}_{\mu} p \rightarrow \mu^{+} p \pi^{-}$(e), and $\bar{\nu}_{\mu} n \rightarrow \mu^{+} n \pi^{-}$(f) measured at ANL 1973 [62], 1979 [63], 1982 [64], BNL 1986 [65], FNAL 1978 [66,67] CERN BEBC 1983 [68], 1984 [69], 1986

Refs $[52,55]$. The MK model includes several significant extensions beyond the current $1 \pi$ production models in GENIE, most importantly from the proper accounting for interference between resonances and NRB. Rather than taking a strictly empirical approach, the NRB contribution is provided by generalized Born graphs for the $1 \pi$ production based on a chiral SU(2) nonlinear $\sigma$ model according to the Hernández, Nieves, and Valverde (HNV) approach; vector form factors are also updated.

The MK model is the first resonance model implementation in GENIE that fully incorporates the interference effects and predictions for pion angular distributions. Although its main applicability is to low- $W$
[70], and 1990 [71] with cut of $W<2 \mathrm{GeV}$ and with no cut on $W$, in comparison with G18_10b_02_11b tune (red solid line) and MK model (preliminary, magenta dashed line) incorporated in GENIE 3. The error bars are the statistical and systematic errors added quadratically

resonances, the high $W$ behavior is not understood. At this time, it is still under development and must be considered preliminary.

Figure 5 shows the total $\mathrm{CC} 1 \pi$ production cross sections for $\nu_{\mu}$ and $\bar{\nu}_{\mu}$ induced reactions with the bare nucleons as predicted by the G18_10b_02_11b CMC (coming from BS [52] and KLN $[50,51]$ ) and by the MK model [57-60], in comparison with the experimental data using bubble-chambers filled with the hydrogen and deuterium. Data from CERN BEBC [68-71] are used without modification. The data of ANL 1982 [64] and BNL 1986 [65] are reanalysed in Ref. [72]. The data of CERN BEBC 1990 [71] with the cut $W<2$ $\mathrm{GeV}$ are revised in Ref. [73]. The data of ANL 1973 
[62] and FNAL $1978[66,67]$ with the cut $W<2 \mathrm{GeV}$ are obtained as the cross sections of $\Delta$ production.

\section{$3.5 \mathrm{COH}$ gamma}

Neutral-current photon emission reactions with nucleons and nuclei are important backgrounds for $\nu_{\mu} \rightarrow$ $\nu_{e}\left(\bar{\nu}_{e} \rightarrow \bar{\nu}_{e}\right)$ appearance oscillation experiments where electromagnetic showers instigated by electrons (positrons) and photons are hard to distinguish. For example, it has implications for the $\mathrm{T} 2 \mathrm{~K}$ oscillation analyses $[74,75]$. In the few-GeV region, these reactions are dominated by the weak excitation of the $\Delta(1232)$ resonance and its subsequent decay into $N$ and $\gamma$ and this process has been available in GENIE since version 2. The coherent reaction channel ( $\mathrm{COH}$ gamma), where the nucleus returns to its ground state after emitting a gamma ray, has a small (5 to 50 times smaller than incoherent photon emission, depending on the neutrino energy) but sizable contribution particularly in the forward direction. For this reason, it is a background for some of the BSM candidates to explain the MiniBooNE anomaly [76] (see also Sect. 4.2).

In spite of its interest, the coherent excitation leading to a gamma production was missing and it has been included following the theoretical development of Ref. [77] but introducing some simplifications to make event generation feasible [78]. Within a microscopic approach, the nuclear current is obtained by summing the contributions of all nucleons. In this sum, the nucleon wave functions remain unchanged leading to nuclear density distributions and nuclear form factors. In the GENIE implementation, empirical parametrisations [79] have been adopted for these form factors, adapted to any nucleus by means of interpolation. The total cross section for the coherent gamma production reaches a plateau for neutrino energies around $2 \mathrm{GeV}$, see Fig. 6 to see examples of integrated cross sections.

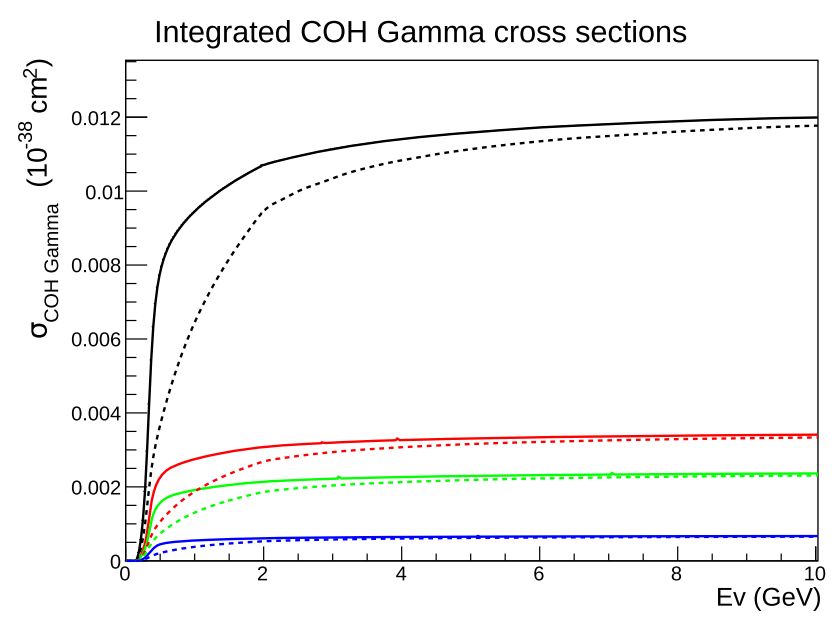

Fig. 6 Integrated cross section of $\mathrm{COH}$ Gamma production from $\nu_{\mu}$ (continuous lines) and $\bar{\nu}_{\mu}$ (dotted lines) on ${ }^{12} \mathrm{C}$ (blue), ${ }^{40} \mathrm{Ar}$ (green), ${ }^{56} \mathrm{Fe}$ (red) and ${ }^{208} \mathrm{~Pb}$ (black)
The development is on target to be released in version v3.2.2 including only the dominant $\Delta$ resonance contribution. The contributions from heavier baryon resonances, which is a correction of the $10 \%$ order sizable for (anti)neutrino energies above $1.5 \mathrm{GeV}[78]$ and potentially relevant for BSM searches at MINERvA, will be released at a later stage.

\subsection{Final state interactions}

Final state interaction (FSI) models are a difficult but important part of the code. Any hadrons produced in principal interaction models are then processed by one of the FSI codes. As a result, any evidence of the principal interaction is masked as hadrons propagate through the residual nucleus. From the beginning, GENIE included the hA model which is a data-driven code that is fully reweightable. With version 3.0, the $\mathrm{hN}$ (full cascade very similar to NuWro and NEUT) was added. With v3.2, we add the INCL $++[3,80]$ and Geant4 extended Bertini [4] models. These are added as libraries with appropriate interfaces. Implementation mainly required a transfer of variables between codes so that the GENIE output would be as close as possible to the native FSI codes. GENIE assumes the Monte Carlo method of choosing interactions as the particle propagates according to the mean free path which depends on position and energy. Both codes assume the hadron was in an incident beam and adjustments were required. For INCL, each hadron coming out of a principal interaction was separately propagated starting onshell. Geant4 unfortunately has all particles interact and then normalizes to template hadron-nucleus cross sections. This is incompatible with lepton production processes and was covered by having the hadrons use the same stepping process as $\mathrm{hA}$ and $\mathrm{hN}$. Although this means the total reaction cross section is different than what the Geant4 code would calculate, the overall results in GENIE are a reasonable description of hadron-nucleus data. A separate article [81] compares $\mathrm{hA}, \mathrm{hN}$, and INCL with other event generators for total reaction cross section and transparency. The conclusions there are similar to what is seen here.

Each of the newer models add significant capabilities. All of the newer codes are based on free hadron-nucleon cross sections with corrections to account for the surrounding nucleons. $\mathrm{hN}$ has medium corrections for both pions [82] and protons [83] and steps particles in space. In the released tunes, the nuclear model is consistent with the one used in the primary interactions. The original hA model tracks particles in the same way as $\mathrm{hN}$ except that it does not have pion medium corrections. INCL $[3,80]$ and Geant4 [4] use a series of shells at different radius, each having a custom depth. Therefore, both naturally include medium and binding energy corrections in a basic way.

Each of the codes includes charge exchange and inelastic scattering, absorption (pions) and knockout (nucleons and kaons), and pion production processes. Tracking of nucleons and pions is common to all codes, 

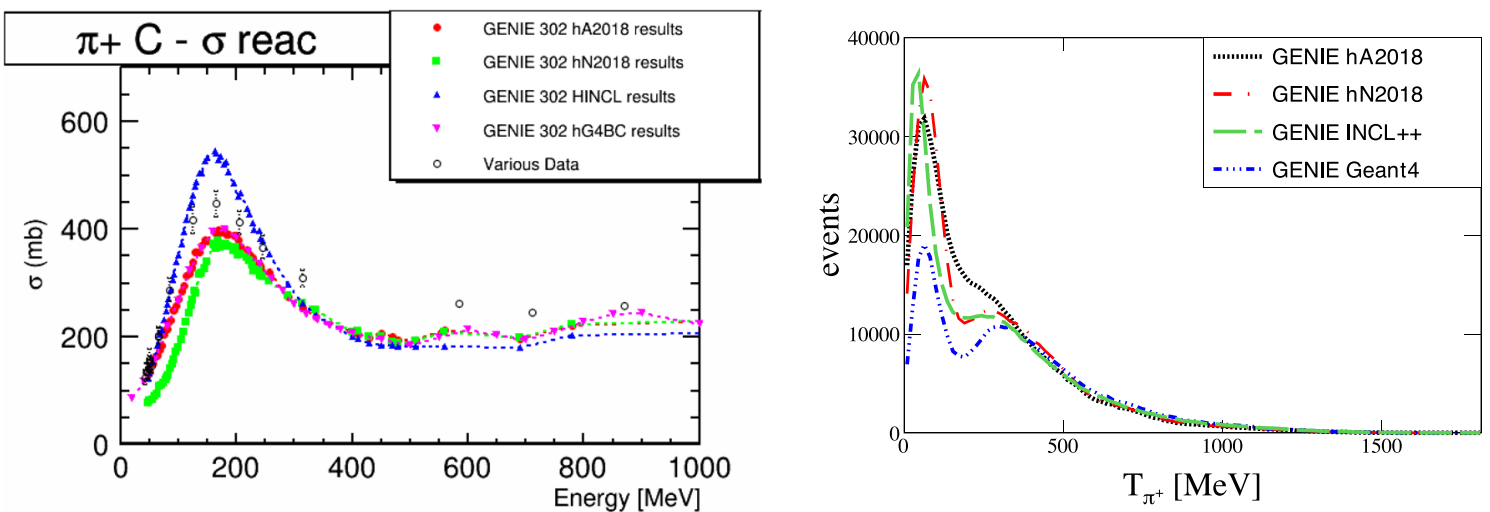

Fig. 7 Distributions for $\pi^{+}$, total $\pi^{+}$reaction cross section for carbon compared to world data [84] (left) and inclusive $\pi^{+}$kinetic energy distribution from $2 \mathrm{GeV} \nu_{\mu}{ }^{40} \mathrm{Ar}$ (right). In each case, results from all 4 models described in the text are shown

but INCL has no additional capabilities. $\mathrm{hA}$ and $\mathrm{hN}$ also treat $K^{+}$interactions and although Geant4 adds a host of additional particles, only kaons are presently enabled. Although, both hA and hN models have simple mechanisms to produce the well-known rise in nucleon yield at energies less than $\sim 20 \mathrm{MeV}$, INCL and Geant4 add the capability to simulate low energy compound nuclear processes and coalescence which adds light ions and photons to the final state.

Despite the wide range of approximations, all 4 models have similar general ability to describe data at higher energies (kinetic energy larger than $\sim 300 \mathrm{MeV}$ ), but show significant variations where nuclear effects are important. Figure 7 left shows the total reaction cross section for $\pi^{+}$-carbon. More important properties of hadrons can be seen in a simulation of $2 \mathrm{GeV} \nu_{\mu}$-argon with all CC interactions enabled. Since each simulation included 2 million events using the same set of principal interactions, these distributions can be compared directly as cross sections. The pion kinetic energy spectrum (Fig. 7 right) is very similar for all models at energies above $300 \mathrm{MeV}$. However, there are significant differences around the peak of the $\Delta(1232) P_{33}$ resonance, showing affects beyond what can be seen in Fig. 7. Although, the kinetic energy distribution (Fig. 8 left) is more similar among the models, strong deviations are seen at low energy. On the other hand, the neutron multiplicities (Fig. 8 right) and low energy proton and neutron kinetic energy spectra (Fig. 9) show wide variation according to model. Both come from the sensitivity to low energy modeling. Both INCL ++ and Geant4 have more sophisticated nuclear models in the initial state and this is one place where INCL and Geant4 show significant advantage. Protons should be suppressed compared to neutrons at low energy by Coulomb effects and the newer models show this. The other significant advantage is in the emission of photons and light ions. The photon spectrum is shown in Fig. 10. Accounting for photons from the excited states in all nuclei requires a very effort. Instead, both INCL and Geant4 use statistical models in the residual nuclei and produce smooth spectra according to the energy allotted to photons.

\subsection{HEDIS}

Historically, the focus of neutrino interaction modelling in GENIE was the medium neutrino energy range (a hundred $\mathrm{MeV}$ up to a hundred $\mathrm{GeV}$ ) relevant for atmospheric neutrino studies, as well as for studies of accelerator-made neutrinos both at short and long baseline experiments. GENIE has the mission to support the global experimental neutrino program and the emergence of the field of high-energy neutrino astronomy [85-87], as well as the FASER $\nu$ [88] and SHiP [89] projects at CERN, generated the demand for accurate GENIE simulations of high energy neutrino interactions, beyond what was available through extrapolations of its model geared towards medium energies. To address this demand, a new HEDIS GENIE package was created [90], implementing high-energy cross section calculation and event generation modules. A new series of new CMC (GHE19_00a, GHE19_00b and GHE19_00d) using alternative HEDIS configurations were constructed. These new CMCs can be applied strictly for neutrino energies above $100 \mathrm{GeV}$, and have been tested up to $1 \mathrm{EeV} .^{5}$

The current HEDIS package includes several scattering mechanisms relevant for high energy neutrinos: deep inelastic scattering (DIS) off nucleons and subleading resonant DIS effects due to neutrino interactions with the photon field of the nucleon [90]. Where possible, changes were implemented through a new generalised interface for structure function calculations. Generally, for DIS scattering off gluons and quarks, in the perturbative regime, the structure functions $F_{i}^{\nu N}$ factorise in terms of process-dependent coefficients $C_{i, a}^{\nu}$ and process-independent PDFs $f_{a}^{N}$ as follows

$$
F_{i}^{\nu N}\left(x, Q^{2}\right)=\sum_{a=g, q} \int_{x}^{1} \frac{d z}{z} C_{i, a}^{\nu}\left(\frac{x}{z}, Q^{2}\right) f_{a}^{N}\left(z, Q^{2}\right)
$$

\footnotetext{
5 Small-x resummation effects are relevant for $\mathrm{E}>1 \mathrm{EeV}$ [91] and they are not included in these CMCs.
} 


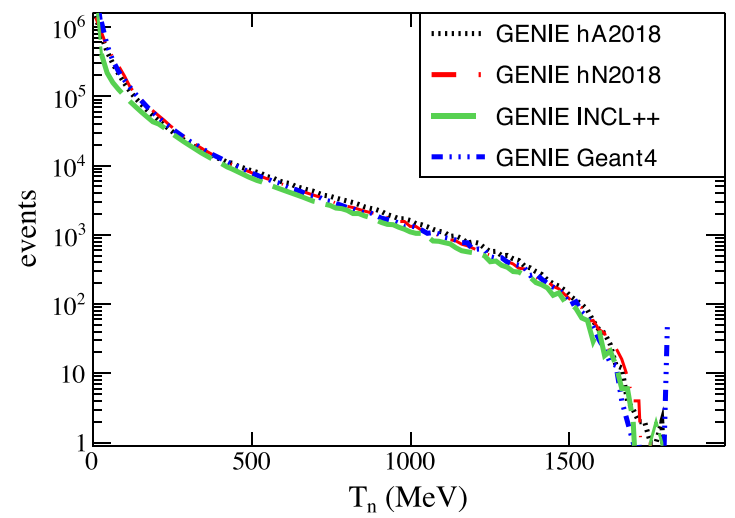

Fig. 8 Neutron distributions from a simulation of $2 \mathrm{GeV} \nu_{\mu}$ case, results from all 4 models described in the text are shown

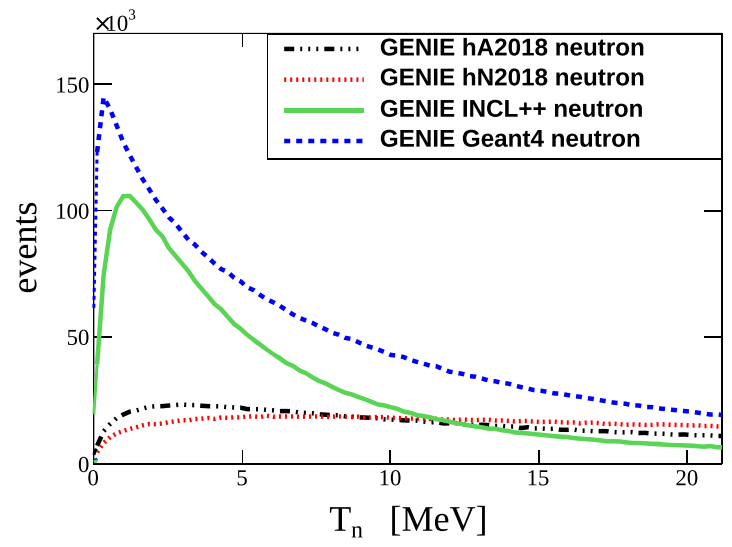

Fig. 9 Left: Neutron (left) and proton (right) kinetic energy distributions from a simulation of $2 \mathrm{GeV} \nu_{\mu}{ }^{40} \mathrm{Ar}$ focusing on low energy responses, in each case, results from

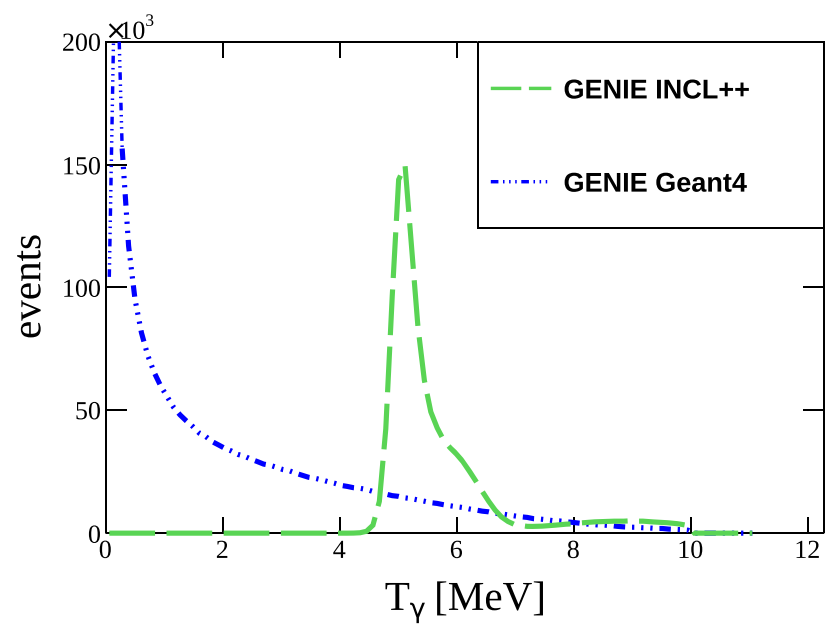

Fig. 10 Photon kinetic energy distributions from a simulation of $2 \mathrm{GeV} \nu_{\mu}{ }^{40} \mathrm{Ar}$ focusing on low-energy responses for INCL and Geant4. The gamma mode is not included in either $\mathrm{hN}$ or hA models

where the coefficients $C_{i, a}^{\nu}$ can be computed in perturbation theory as a power expansion in the strong coupling constant $\alpha_{s}$. The evolution of PDFs is deter-

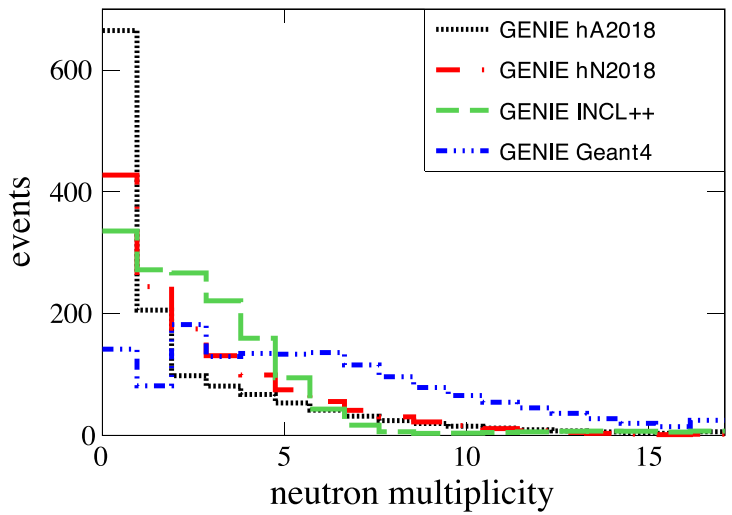

${ }^{40} \mathrm{Ar}$, kinetic energy (left) and multiplicity (right). In each

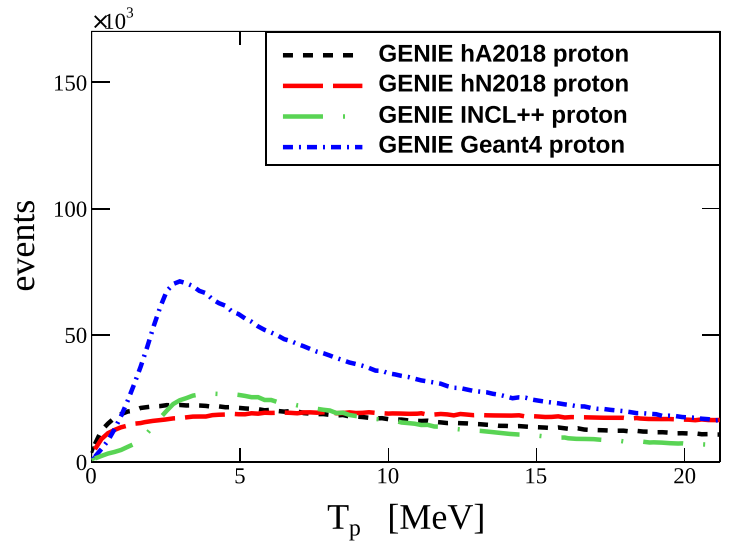

all 4 models described in the text are shown. Results from INCL ++ and Geant4 models described in the text are shown

mined by the DGLAP equations and structure functions are computed using the APFEL program [91]. The main NLO DIS calculation implemented in HEDIS is the BGR18 [92] model (GHE19_00a). In the BGR18 calculation, all inputs are at NLO accuracy. PDF sets are taken from the NNPDF3.1sx [93] global analysis of collider data, incorporating (through PDF reweighting) the impact of $\mathrm{LHCb} D$-meson production in $p p$ collisions (small- $x$ PDF constraints beyond the kinematic range of HERA data) [94-96]. The calculation is using the FONLL scheme [97] to account for quark mass effects. In addition to BGR18, the CMS11 [98] and GGHR20 [90] NLO DIS calculations is also implemented in HEDIS for reference (GHE19_00b and GHE19_00d, respectively). Figure 11 shows the prediction of the total cross section per nucleon for $\nu_{\mu} \mathrm{CC}$ scattering for the three models described above. The relative rise of the CMS11 calculation in the low- $E$ region is due to the inclusion of low-momentum contributions $(1.0<Q<1.64 \mathrm{GeV})$ which are absent in BGR18 and GGHR20. Besides deep inelastic scattering (DIS), HEDIS incorporates contributions from coherent scattering from the nucleus, which represents a substantial (5-10\%) contribution for heavy nuclei [90]. Glashow scattering, which is simulated externally to HEDIS, is 


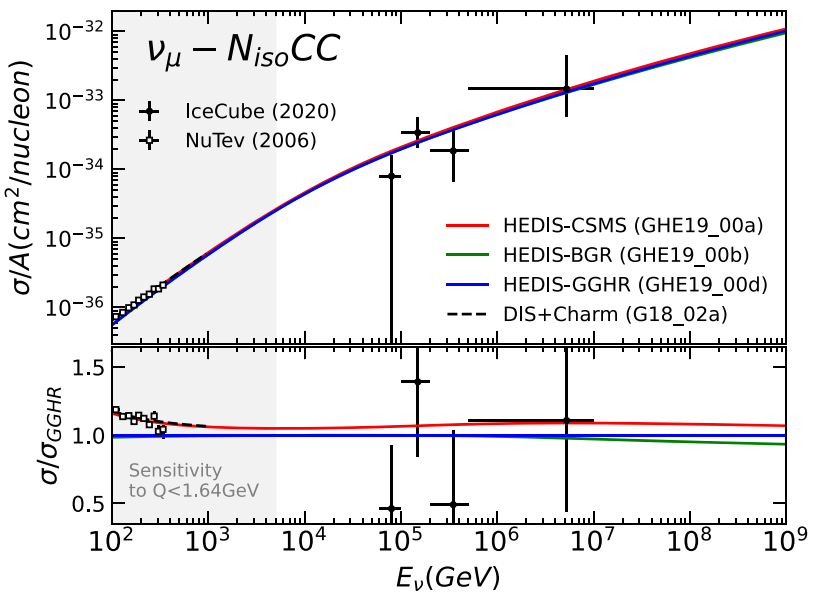

Fig. 11 Predictions of total cross section per nucleon for $\nu_{\mu}$ CC scattering assuming isoscalar target for three different models implemented in HEDIS: BGR18 [92], CMS11 [98] and GGHR20 [90]. Predictions from G18_02a (assuming DIS and charm production) and $\mathrm{NuTeV}[100]$ and IceCube [101] measurements are shown up to $E_{\nu}=1 \mathrm{TeV}$ for comparison. Grey area shows the region where low-momentum contributions $(Q<1.64 \mathrm{GeV})$ are relevant

also incorporated in the high energy GENIE CMCs. As part of the HEDIS development, an alternative interface to PYTHIA6 which originates from LEPTO [99] was installed in GENIE. A comparative analysis and consolidation of the two PYTHIA/GENIE interfaces will be the subject of a future development project.

The DIS model used in the medium energy CMCs is based on the Bodek-Yang formalism, which describes the low $Q^{2}$ region better than $\mathrm{pQCD}$ models. However, it does not account for heavy sea quarks, which are relevant at high energies. In the region where low $Q^{2}$ and heavy quarks contributions are negligible (between 0.1 and $1 \mathrm{TeV}$ ), the two formalisms agree as shown in Fig. 11. ${ }^{6}$ Joining up the medium and high energy simulations into CMCs that span the full energy range will be the objective of a future development project.

\subsection{Coherent elastic neutrino-nucleus scattering}

At energies below $100 \mathrm{MeV}$, neutrino interactions with complex nuclei are dominated by coherent elastic neutrino-nucleus scattering ( $\mathrm{CE} \nu \mathrm{NS})$, a neutralcurrent reaction in which the final nucleus is left in its ground state. Since the only experimental signature is the small nuclear recoil kinetic energy, direct detection of $\mathrm{CE} \nu \mathrm{NS}$ events is challenging. Despite being anticipated theoretically several decades ago [102], only two measurements $[103,104]$ have been reported to date, both by the COHERENT experiment at Oak Ridge National Laboratory. Due to the usefulness of precision

6 A better agreement is found with CSMS because the PDFs used in this configuration have a lower $Q$ boundary $\left(Q_{\min }=1 \mathrm{GeV}\right)$ than the PDFs used in BGR and GGHR $\left(Q_{\min }=1.64 \mathrm{GeV}\right)$.

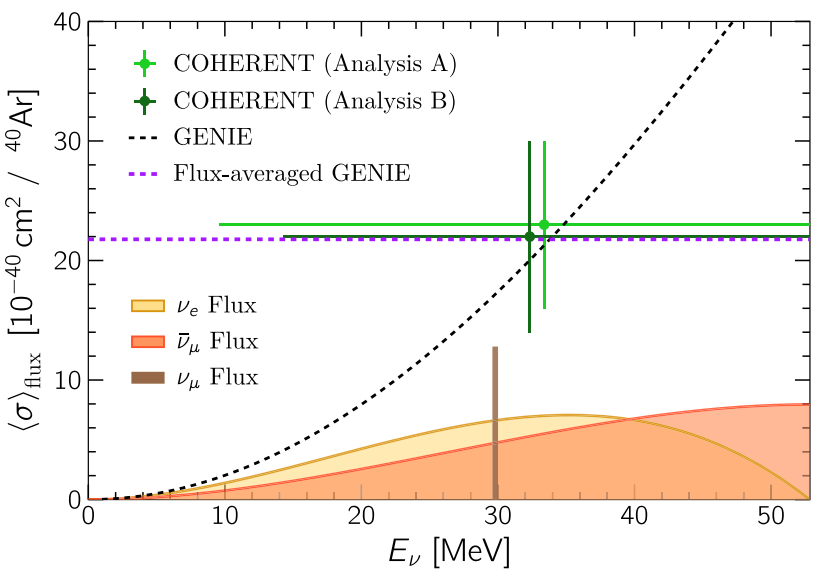

Fig. 12 GENIE energy-dependent (dashed black) and flux-averaged (dashed violet) total cross section for coherent elastic neutrino-nucleus scattering on ${ }^{40} \mathrm{Ar}$ compared to data from the COHERENT experiment (green points). The neutrino flux spectra for the three participating neutrino species are also shown with arbitrary normalization

$\mathrm{CE} \nu \mathrm{NS}$ data for studying nuclear structure $[105,106]$, searching for physics beyond the Standard Model [107], and, perhaps, for monitoring reactors [108], this process is the subject of increasing theoretical and experimental attention worldwide.

While proprietary codes are currently used by some experiments to simulate $\mathrm{CE} \nu \mathrm{NS}$, the GENIE v3 implementation represents the first realistic treatment of this process in a widely-distributed neutrino event generator. ${ }^{7}$ In terms of the kinetic energy $T_{A}$ of the final nucleus, the $\mathrm{CE} \nu \mathrm{NS}$ differential cross section is given by

$$
\frac{d \sigma}{d T_{A}}=\frac{G_{F}^{2} M}{4 \pi} F^{2}\left(Q^{2}\right)\left[2-\frac{2 T_{A}}{E_{\nu}}+\frac{T_{A}^{2}-M T_{A}}{E_{\nu}^{2}}\right]
$$

where $M$ is the nuclear mass, $G_{F}$ is the Fermi constant, and $E_{\nu}$ is the incident neutrino energy in the laboratory frame. The nuclear form factor $F\left(Q^{2}\right)$ is sensitive to nuclear structure effects primarily through the neutron density distribution. The few-percent theoretical uncertainties on $F\left(Q^{2}\right)$ were recently studied for ${ }^{40} \mathrm{Ar}$ in Ref. [106]. In GENIE, the form factor calculation currently used is that of Patton et al. [110]. The dashed black line in Fig. 12 shows the GENIE prediction for the CE $\nu$ NS total cross section on ${ }^{40} \mathrm{Ar}$ as a function of neutrino energy. The flux-averaged prediction (dashed violet) is also shown for a pion and muon decay-at-rest neutrino source. Excellent agreement is seen between the fluxaveraged GENIE prediction and the recent COHERENT measurement [104] (green points).

\footnotetext{
7 The open-source MARLEY generator [109] provides a $\mathrm{CE} \nu \mathrm{NS}$ model which relies on a rough approximation: the $Q^{2}$ dependence of the nuclear form factor is completely neglected.
} 


\subsection{New comprehensive model configurations and tunes}

The CMCs supported by the collaboration are natural evolutions of the GENIE v2 default configuration when a variety of roughly equal models for the same process are available. Care is taken to group compatible models together to maintain theoretical consistency and our goal of reproducing theoretical models accurately. CMCs are often grouped so that a full set of interactions is available for neutrino energies $100 \mathrm{MeV}-$ $1 \mathrm{TeV}$.

The first group of CMCs is historically motivated: it is based on the default configuration in previous releases and simply provides updates for processes that were introduced later, like $\Lambda$ production or diffractive scattering from free protons. All these CMC IDs start with G18 01.

The second family is an improvement of the first group in terms of the resonance model. Specifically, improvements of the Rein-Sehgal resonance models for $\mathrm{CC}$ and NC neutrino-production, as well as for CC and $\mathrm{NC}$ coherent production of mesons, were replaced with updated models by Berger and Sehgal [52]. Their corresponding CMC are the G18_02.

The third family, G18_10, was constructed aiming to deliver the most up to date theoretical nuclear model simulations. With respect to G18_02 CMCs, the $0 \pi$ production models (Llewellyn Smith CC quasielastic and GENIE's empirical multinucleon model) are replaced with implementations of the corresponding Valencia models by Nieves et al. [39]. Within this group of models, the nuclear environment is modelled using a Local Fermi Gas, matching the inputs used for the published Valencia calculations. In the same groups belongs the CMCs that have the $0 \pi$ production models based on the SuSAv2 approach, and they are labeled G21_11 (for neutrinos) and GEM21_11 (for electrons).

Out of these main ideas, a number of CMCs can be constructed simply changing more detailed aspects like FSI or form factors. To indicate the FSI, one more letter is added to the CMC name: a for $\mathrm{hA}, \mathrm{b}$ for $\mathrm{hN}$, c for INCL, $\mathrm{d}$ for GEANT.

The complete tune names contain 2 additional fields that identify the tune performed using a CMC. All fields must be specified, but the simplest choice is to use the same tune as in v2 by adding zeroes, e.g. G18_02a_00_000. More recent examples use the postfix _02_11a or _02_11b that denote a tune against neutrino pion production data on protons and deuterium targets: specifically _02_11b identifies the tune described in [2]. Other notable examples are the hadronisation tunes described in [111] that have postfix _03_330 or _03_320 depending on the data used in the fit.

Figure 13 compares recent neutrino cross section data to theoretical predictions generated using several different GENIE CMCs. The left panel shows the fluxaveraged differential cross section obtained by MicroBooNE for the reconstructed muon scattering cosine in pionless $\nu_{\mu} \mathrm{CC}$ events containing at least one final state proton [112]. The three CMCs compared to the data are
G18_01a_00_000 (dashed blue), G18_10a_02_11b (dotted violet) and G21_11b_00_000 (solid green). According to our naming scheme, the first is the historically motivated CMC that uses hA FSI, the second is the theory motivated tuned version, also using hA FSI, and the last is the latest implemented CMC that uses SuSAv2 with the hN FSI. Substantially improved agreement is achieved by G18_10a_02_11b at forward angles, which is driven especially by the Valencia model's RPA-based treatment of long-range nucleon correlations. The right panel of Fig. 13 shows a similar comparison to a measurement by the MINER $\nu$ A Collaboration of single $\pi^{-}$ production in $\mathrm{CC} \bar{\nu}_{\mu}$ scattering on hydrocarbon [113]. In this case, the choice of CMCs in the comparison emphasizes differences in the RES model and FSIs. G18_01a_00_000 (dashed blue) and G18_02a_00_000 (dotted violet) share the same hA model for FSIs but use the Rein-Sehgal [46] and Berger-Sehgal [52] treatments, respectively, to describe RES interactions. Two additional CMCs are shown in which the Berger-Sehgal model is also used. G18_02a_02_11b employs exactly the same physics models as G18_02a_00_000, but a number of parameters have been tuned based on fits to neutrinonucleon scattering data [2]. In G18_02b_02_11b, the same tuned parameters are adopted, but the FSI model has been switched from hA to hN (see Sect.3.6 for details). Although the other CMC differences in the comparison play some role, the improved agreement seen when using the tuned CMCs is the most significant effect.

\section{Beyond the standard model event generators in GENIE}

Searches for physics Beyond the Standard Model (BSM) form an important pillar of the science program of current and future neutrino experiments. New detectors with unprecedented imaging capabilities, both massive ones at deep underground locations and smaller ones in near proximity to very intense proton and neutrino beams, create opportunities for expanding the sensitivity of established BSM searches and perform novel ones: Searches for nucleon decay, $n-\bar{n}$ oscillations, deviations from the SM neutrino trident rates, millicharged particles, dark neutrinos, light/boosted dark matter are, to name a few, some of improved or new BSM searches that will be carried out. Standard neutrino interactions are a background to BSM searches and, therefore, it is important to simulate both BSM and neutrino interactions in a common physics framework using, for example, common nuclear and intranuclear hadron transport modelling. GENIE supports these searches with a full implementation of four BSM generators: Generators for nucleon decay and $n-\bar{n}$ oscillations have been available in GENIE for several years. Recent additions, in GENIE v3 series, include the addition of a full Boosted Dark Matter (BDM) generator, and well as a first ver- 

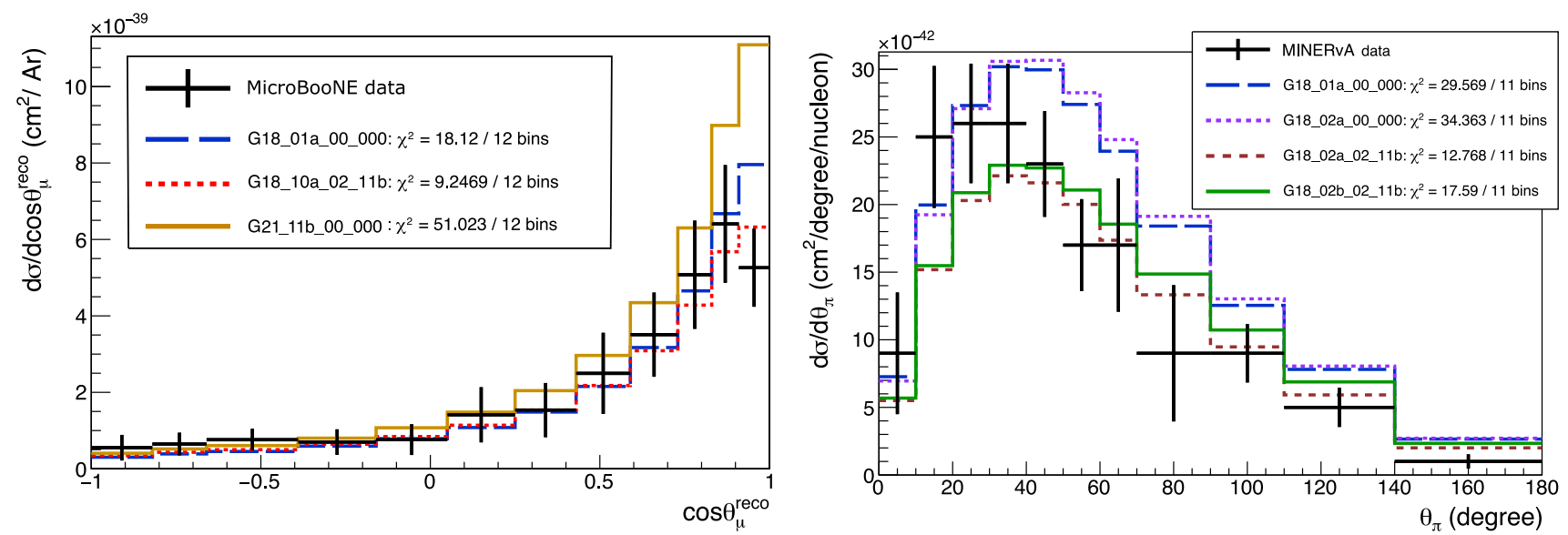

Fig. 13 Comparisons of representative GENIE v3 CMC predictions to recent neutrino cross section measurements obtained by the MicroBooNE (left) and MINER $\nu$ A (right) experiments. See the text for the dataset descriptions

sion of Dark Neutrino generator geared for low energy experiments.

\subsection{Boosted dark matter}

A BDM generator was made available in GENIE with the release of v3.0.0. The generator covers a extensive class of physics models described by the interaction Lagrangian

$$
\mathcal{L}_{\text {int }}=g_{Z^{\prime}} Z_{\mu}^{\prime} J_{Z^{\prime}, \psi}^{\mu}
$$

where

$$
J_{Z^{\prime}, \psi}^{\mu}=\bar{\psi} \gamma^{\mu}\left(Q_{L}^{\psi} P_{L}+Q_{R}^{\psi} P_{R}\right) \psi
$$

and $\psi=\chi, u, d, s, c, e$. The model is specified by charges $Q_{L R}^{\psi}$, the gauge coupling $g_{Z^{\prime}}$ and the masses of the dark matter particle $\chi$ and of the mediator $Z^{\prime}$.

A substantial upgrade of the BDM generator was deployed in GENIE 3.2.0, aligning it with the model described in Ref. [114]. The upgrade allows for a broader set of particle physics models which may incorporate both vector and axial couplings as well as different isospin structures. The upgraded generator also enables simulations involving new probes (anti-darkmatter), a new target (scattering off electrons), and an improved model of the elastic scattering process which includes a pseudoscalar form factor.

\subsection{Dark neutrino generator}

Dark neutrino interactions arise from an extension of the SM Lagrangian adding a fourth neutrino flavour that mixes with the SM neutrinos [115]. This extension can explain the low energy electromagnetic (EM) excess detected by short baseline experiments. These new dark neutrinos are relatively heavy $(\mathrm{O}(100) \mathrm{MeV})$. This extension comes with a new light neutral boson (lighter than the dark neutrino) that couples with both EM and weak charge, although the coupling with the weak charge is considered negligible as shown from model developers' fits. The new Lagrangian predicts a dark equivalent for every existing NC SM interaction. In these new interactions, instead of exchanging a $Z$ boson, the incoming neutrino will exchange the new light boson and produce an on-shell dark neutrino in the final state. The dark neutrino will later decay into either SM neutrinos or electron pair. At the moment only the dominant interaction is implemented: the COH Dark (Quasi) elastic interaction, which is the dark equivalent of $\mathrm{CE} \nu \mathrm{NS}$. The implemented cross section was given to us by Pedro Machado, one of the model's authors. Details of the implementation, including the differential cross section, can be found in a GENIE public note [116].

The model depends on several parameters: the masses of the dark particles $\left(M_{N}\right.$ dark neutrino and $M_{Z_{D}}$ mediator), the neutrino mixing and the coupling between the dark boson and the EM charge. All these parameters affects the cross section: some just the intensity (mixings and $\alpha_{D}$ ) while the masses control the production threshold and the way that the cross section decreases as a function of $Q^{2}$. Example of different parameters configurations can be seen in Fig. 14.

\section{Summary}

As neutrino physics continues to move into its precision era, the need for high-quality simulations of neutrinonucleus scattering and related processes will only continue to grow. GENIE has already been a standard tool in the field for many years, forming an indispensable part of many experiments' simulation workflows and offering a historical default model which has been widely tested against neutrino cross-section data. Major version 3 of GENIE builds upon this foundation with wide-ranging improvements to both physics modeling and to the technical machinery needed for daily use in experimental analyses. 
$\mathrm{COH}$ Dark neutrino production

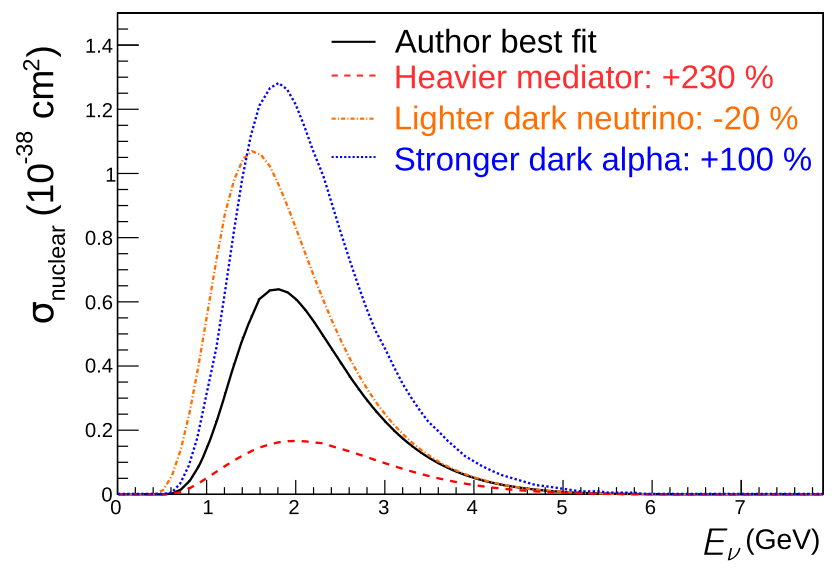

Fig. 14 Integrated cross section for $\mathrm{COH}$ dark neutrino scattering from $\nu_{\mu}$ on Ar for different parameters. The values used for the black curve are the one obtained from the MiniBooNE excess fit by the model authors in [115] and they are: $M_{N}=420 \mathrm{MeV}, M_{Z_{D}}=30 \mathrm{MeV}$ and $\alpha_{D}=0.25$. Other curves varies one parameter at a time, according to the legend: the new values are selected purely for plotting purposes

A key technical addition in version 3 is the concept of a comprehensive model configuration, which allows multiple curated sets of compatible physics models to coexist in GENIE with a user-friendly means of switching between them. The new event library interface allows an external generator's physics models to be used within the GENIE framework while respecting the integrity of both codes. When these features are combined with a growing global analysis of neutrino scattering data and an advanced toolset for parameter tuning and uncertainty quantification, they form a stateof-the-art platform for meeting the simulation needs of current and future experiments.

The GENIE collaboration and external contributors continue to improve all aspects of the code's physics models, from the description of the nuclear target to hadronic final-state interactions, and including both standard and BSM processes. Improving the quality of GENIE's electron scattering mode and its consistency with the neutrino cross-section implementations has been an area of recent emphasis. The v3.2 release features various enhancements to GENIE's simulation capabilities for accelerator neutrinos, including entirely new model implementations for $\mathrm{QE}$ and $2 p 2 h$ (SuSAv2), single pion production (MK), and intranuclear hadron transport (INCL and Geant4). These core physics topics are complemented by substantial developments at lower (CEvNS) and higher (HEDIS) energies, reflecting the collaboration's mission to provide truly universal neutrino interaction modeling.

Acknowledgements We would like to thank the CCIN2P3 Computing Center, as well as the Particle Physics Department at Rutherford Appleton Laboratory for pro- viding computing resources and for their support. This work, as well as the ongoing development of several other GENIE physics tunes, was enabled through a $\mathrm{PhD}$ studentship funded by STFC through LIV.DAT, the Liverpool Big Data Science Centre for Doctoral Training (project reference: 2021488). The initial conceptual and prototyping work for the development of the GENIE / Professor interfaces, as well as for the development of the GENIE global analysis framework that, currently, underpins several analyses, was supported in part through an Associateship Award by the Institute of Particle Physics Phenomenology, University of Durham. This document was prepared by the GENIE collaboration using the resources of the Fermi National Accelerator Laboratory (Fermilab), a U.S. Department of Energy, Office of Science, HEP User Facility. Fermilab is managed by Fermi Research Alliance, LLC (FRA), acting under Contract No. DE-AC02-07CH11359. The authors acknowledge support from US Department of Energy under contract No. DE-SC0007914. A.P. acknowledges support from the Visiting Scholars Award Program of the Universities Research Association. A.G. acknowledges support from the European Union's H2020-MSCA Grant Agreement No. 101025085. L.A.R. has been supported by the Spanish Ministerio de Ciencia e Innovación and the European Regional Development Fund (ERDF) under contract FIS2017-84038C2-1-P, the EU STRONG-2020 project under the program H2020-INFRAIA-2018-1, grant agreement no. 824093 and by Generalitat Valenciana under contract PROMETEO/2020/023. I.D.K. acknowledges support from Russian Science Foundation grant No. 18-12-00271. The GENIE code comes from the work of many people beyond the authors of this article and we are grateful to them. They include Josh Berger, Yanou Cui, Lina Necib, Yun-Tse Tsai, and Yue Zhao (boosted dark matter); Iker de Icaza and Pedro Machado (dark neutrino); Joe Johnston (Valencia QE model); Tomasz Golan (Oset cascade model); Jarek Nowak (Berger Sehgal baryon resonance model); Jackie Schwehr, Dan Cherdack, and Rik Gran (Valencia $2 p 2 h$ model); Jon Sensenig, Eduardo Saul-Sala, and Kathryn Sutton (coherent gamma); Chris Backhouse (event library); Stephen Dolan and Guillermo Megias (SuSAv2); and Dennis Wright and Makoto Asai (Geant4).

Open Access This article is licensed under a Creative Commons Attribution 4.0 International License, which permits use, sharing, adaptation, distribution and reproduction in any medium or format, as long as you give appropriate credit to the original author(s) and the source, provide a link to the Creative Commons licence, and indicate if changes were made. The images or other third party material in this article are included in the article's Creative Commons licence, unless indicated otherwise in a credit line to the material. If material is not included in the article's Creative Commons licence and your intended use is not permitted by statutory regulation or exceeds the permitted use, you will need to obtain permission directly from the copyright holder. To view a copy of this licence, visit http://creativecomm ons.org/licenses/by/4.0/. 


\section{References}

1. C. Andreopoulos et al., The GENIE Neutrino Monte Carlo Generator. Nucl. Instrum. Methods A 614(1), 87-104 (2010). https://doi.org/10.1016/j.nima.2009. 12.009. arXiv:0905.2517 [hep-ph]

2. J. Tena-Vidal et al. Neutrino-nucleon cross-section model tuning in GENIE v3. arXiv:2104.09179 [hep-ph]

3. D. Mancusi et al., Improving the description of proton-induced one-nucleon removal in intranuclearcascade models. Phys. Rev. C 91(3), 034602 (2015). https://doi.org/10.1103/PhysRevC.91.034602. arXiv:1411.4562 [nucl-th]

4. D.H. Wright, M.H. Kelsey, The Geant4 Bertini Cascade. Nucl. Instrum. Methods A 804, 175-188 (2015). https://doi.org/10.1016/j.nima.2015.09.058

5. A. Buckley et al., Systematic event generator tuning for the LHC. Eur. Phys. J. C 65(12), 331-357 (2010). https://doi.org/10.1140/epjc/ s10052-009-1196-7. arXiv:0907.2973 [hep-ph]

6. SVN webiste. https://subversion.apache.org/

7. Git webiste. https://git-scm.com/

8. P.A. Zyla et al., Review of particle physics. PTEP 2020(8), 083C01 (2020). https://doi.org/10.1093/ ptep/ptaa104

9. H. Schulz et al. Professor webiste. https://professor. hepforge.org

10. B. Chris. The GENIE event library generator interface. Talk delivered at the NuSTEC Workshop: New Directions in Neutrino-Nucleus Scattering, 15 March 2021. https://indico.phys.vt.edu/event/44/ contributions/901/

11. C. Andreopoulos et al. The GENIE Neutrino Monte Carlo Generator: Physics and User Manual. arXiv:1510.05494 [hep-ph]

12. P.A.N. Machado, O. Palamara, D.W. Schmitz, The short-baseline neutrino program at Fermilab. Ann. Rev. Nucl. Part. Sci. 69, 363-387 (2019). https://doi.org/10.1146/annurev-nucl-101917-020949. arXiv:1903.04608 [hep-ex]

13. K. Abe et al. Hyper-Kamiokande Design Report. arXiv:1805.04163 [physics.ins-det]

14. B. Abi et al., Deep Underground Neutrino Experiment (DUNE), Far Detector Technical Design Report, Volume I. Introduction to DUNE. JINST 15(08), T08008 (2020). https://doi.org/10.1088/1748-0221/ 15/08/T08008. arXiv:2002.02967 [physics.ins-det]

15. A. Bodek, J.L. Ritchie, Fermi motion effects in deep inelastic lepton scattering from nuclear targets. Phys. Rev. D 23(5), 1070-1091 (1981). https://doi.org/10. 1103/PhysRevD.23.1070

16. O. Hen et al., Nucleon-nucleon correlations, shortlived excitations, and the quarks within. Rev. Mod. Phys. 89(4), 045002 (2017). https://doi.org/10.1103/ RevModPhys.89.045002. arXiv:1611.09748 [nucl-ex]

17. J. M. Nieves, J. E. Amaro, M. Valverde. "Inclusive quasi-elastic neutrino reactions". Phys. Rev. C 70(5) (2004). [Erratum: ibid. 72.1 (2005) p. 019902], p. 055503. https://doi.org/10.1103/PhysRevC. 70.055503, https://doi.org/10.1103/PhysRevC.72. 019902. arXiv:nucl-th/0408005

18. K.S. Egiyan et al., Measurement of 2- and 3-nucleon short range correlation probabilities in nuclei. Phys.
Rev. Lett. 96(8), 082501 (2006). https://doi.org/10. 1103/PhysRevLett.96.082501. arXiv:nucl-ex/0508026

19. A. Papadopoulou et al., Inclusive electron scattering an the GENIE Neutrino Event Generator. Phys. Rev. D 103(11), 113003 (2021). arXiv:2009.07228 [nucl-th]

20. R. Bradford et al., A new parameterization of the nucleon elastic form factors. Nucl. Phys. B (Proc. Suppl.) 159, 127-132 (2006). https://doi.org/10.1016/ j.nuclphysbps.2006.08.028. arXiv:hep-ex/0602017

21. P.E. Bosted, M.E. Christy, Empirical fit to inelastic electron-deuteron and electron-neutron resonance region transverse cross-sections. Phys. Rev. C $\mathbf{7 7}(6)$, 065206 (2008). https://doi.org/10.1103/PhysRevC.77. 065206. arXiv:0711.0159 [hep-ph]

22. M.E. Christy, P.E. Bosted, Empirical fit to precision inclusive electron-proton cross- sections in the resonance region. Phys. Rev. C 81(5), 055213 (2010). https://doi.org/10.1103/PhysRevC.81.055213. arXiv:0712.3731 [hep-ph]

23. O. Lalakulich, U. Mosel, GiBUU and shallow inelastic scattering. AIP Conf. Proc. 1663(1), 040004 (2015). https://doi.org/10.1063/1.4919474. arXiv:1303.6677 [nucl-th]

24. J. McElwee, "Is T2K missing energy? Searching the electron-scattering data archives for robust removal energy uncertainties". Proceedings of the 22nd International Workshop on Neutrinos from Accelerators (NuFact 2021), Cagliari, Italy (and online), September 6-11, 2021. https://indico.cern.ch/event/ 855372/contributions/4436393/attachments/2305859/ 3922865/nufact-mcelwee.pdf

25. J. Żmuda et al., NuWro Monte Carlo generator of neutrino interactions - first electron scattering results. Acta Phys. Pol. B 46(11), 2329 (2015). https://doi.org/10.5506/APhysPolB.46.2329. arXiv:1510.03268 [hep-ph]

26. T. Katori, Meson exchange current (MEC) models in neutrino interaction generators. AIP Conf. Proc. 1663, 030001 (2015). https://doi.org/10.1063/ 1.4919465. arXiv:1304.6014 [nucl-th]

27. M. Khachatryan, Validation of neutrino energy estimation using electron scattering data. $\mathrm{PhD}$ thesis. Old Dominion U., (2019). https://doi.org/10.2172/ 1768400. https://digitalcommons.odu.edu/physics_ etds $/ 123$

28. A. Ashkenazy et al. Electrons For Neutrinos. Addressing Critical Neutrino-nucleus Issues A Run Group Proposal Resubmission to Jefferson Lab PAC 46. https://www.jlab.org/exp_prog/proposals/ 18/C12-17-006.pdf. C12-17-006

29. D. Achim, Recent results from the Mainz Microtron MAMI and an outlook for the future. AIP Conf. Proc. 1735, 020006 (2016). https://doi.org/10.1063/ 1.4949374

30. A.M. Ankowski et al., Lepton-nucleus cross section measurements for DUNE with the LDMX detector. Phys. Rev. D 101(5), 053004 (2020). https://doi. org/10.1103/PhysRevD.101.053004. arXiv:1912.06140 [hep-ph]

31. C.H.L. Smith, Neutrino reactions at accelerator energies. Phys. Rept. 3(5), 261-379 (1972). https://doi. org/10.1016/0370-1573(72)90010-5 
32. J. Engel, Approximate treatment of lepton distortion in charged current neutrino scattering from nuclei. Phys. Rev. C 57(4), 2004-2009 (1998). https://doi.org/10. 1103/PhysRevC.57.2004. arXiv:nucl-th/9711045

33. T. De Forest, Off-shell electron-nucleon cross sections: The impulse approximation. Nucl. Phys. A 392(2), 232-248 (1983). https://doi.org/10.1016/ 0375-9474(83)90124-0

34. A. Bodek, T. Cai, Removal energies and final state interaction in lepton nucleus scattering. Eur. Phys. J. C 79(4), 293 (2019). https://doi.org/10.1140/epjc/ s10052-019-6750-3. arXiv:1801.07975 [nucl-th]

35. A.A. Aguilar-Arevalo et al., First measurement of the muon neutrino charged current quasielastic double differential cross section. Phys. Rev. D 81(9), 092005 (2010). https://doi.org/10.1103/PhysRevD.81.092005. arXiv:1002.2680 [hep-ex]

36. P. Barreau et al., Deep inelastic electron scattering from Carbon. Nucl. Phys. A 402(3), 515-540 (1983). https://doi.org/10.1016/0375-9474(83)90217-8

37. S. Dolan, G.D. Megias, S. Bolognesi, Implementation of the SuSAv2-meson exchange current 1p1h and $2 \mathrm{p} 2 \mathrm{~h}$ models in GENIE and analysis of nuclear effects in T2K measurements. Phys. Rev. D 101(3), 033003 (2020). https://doi.org/10.1103/PhysRevD. 101.033003. arXiv:1905.08556 [hep-ex]

38. R. González-Jiménez et al., Extensions of superscaling from relativistic mean field theory: the SuSAv2 model. Phys. Rev. C 90(3), 035501 (2014). https://doi.org/10. 1103/PhysRevC.90.035501. arXiv:1407.8346 [nucl-th]

39. J.M. Nieves, I.R. Simo, M.J.V. Vacas, Inclusive charged-current neutrino-nucleus reactions. Phys. Rev. C 83(4), 045501 (2011). https://doi.org/10.1103/ PhysRevC.83.045501. arXiv:1102.2777 [hep-ph]

40. R. Gran et al., Neutrino-nucleus quasi-elastic and 2p2h interactions up to $10 \mathrm{GeV}$. Phys. Rev. D $\mathbf{8 8}(11)$, 113007 (2013). https://doi.org/10.1103/PhysRevD.88. 113007. arXiv:1307.8105 [hep-ph]

41. I.R. Simo et al., Emission of neutron-proton and proton-proton pairs in neutrino scattering. Phys. Lett. B 762, 124-130 (2016). https://doi.org/10.1016/j. physletb.2016.09.021. arXiv:1607.08451 [nucl-th]

42. I.R. Simo et al., Relativistic model of $2 \mathrm{p}-2 \mathrm{~h}$ meson exchange currents in (anti)neutrino scattering. J. Phys. G 44(6), 065105 (2017). https://doi.org/10. 1088/1361-6471/aa6a06. arXiv:1604.08423 [nucl-th]

43. J. Schwehr, D. Cherdack, R. Gran. GENIE implementation of IFIC Valencia model for QE-like $2 \mathrm{p} 2 \mathrm{~h}$ neutrino-nucleus cross section. arXiv:1601.02038 [hep$\mathrm{ph}]$

44. R. Acciarri et al., Detection of back-to-back proton pairs in charged-current neutrino interactions with the ArgoNeuT detector in the NuMI low energy beam line. Phys. Rev. D 90(1), 012008 (2014). https://doi.org/10. 1103/PhysRevD.90.012008. arXiv:1405.4261 [hep-ex]

45. F. Ravndal, Weak production of nuclear resonances in a relativistic quark model. Nuovo Cim. 18 A(3), 385415 (1973). https://doi.org/10.1007/BF02722789

46. D. Rein, L.M. Sehgal, Neutrino-excitation of baryon resonances and single pion production. Ann. Phys. 133(10), 79-153 (1981). https://doi.org/10.1016/ 0003-4916(81)90242-6
47. E.A. Paschos, J.-Y. Yu, Neutrino interactions in oscillation experiments. Phys. Rev. D 65(3), 033002 (2002). https://doi.org/10.1103/PhysRevD.65.033002. arXiv:hep-ph/0107261

48. A. Bodek, I. Park, U. Yang, Improved low $Q^{2}$ model for neutrino and electron nucleon cross sections in few $\mathrm{GeV}$ region. Nucl. Phys. B (Proc. Suppl.) 139, 113-118 (2005). https://doi.org/10.1016/ j.nuclphysbps.2004.11.208. arXiv:hep-ph/0411202

49. A. Bodek, U. Yang, NUFACT09 update to the BodekYang unified model for electron- and neutrino-nucleon scattering cross sections. AIP Conf. Proc. 1222, 233237 (2010). https://doi.org/10.1063/1.3399303

50. K.S. Kuzmin, V.V. Lyubushkin, V.A. Naumov, Lepton polarization in neutrino nucleon interactions. Mod. Phys. Lett. A 19(38), 2815-2829 (2004). https://doi.org/10.1142/S0217732304016172. arXiv:hep-ph/0312107

51. K.S. Kuzmin, V.V. Lyubushkin, V.A. Naumov, Extended Rein-Sehgal model for tau lepton production. Nucl. Phys. B (Proc. Suppl.) 139, 158-161 (2005). https://doi.org/10.1016/j.nuclphysbps.2004. 11.213. arXiv:hep-ph/0408106

52. Ch. Berger, L. M. Sehgal, Lepton mass effects in single pion production by neutrinos. Phys. Rev. D 76(11) (2007). [Erratum: ibid. 77.5 (2008) p. 059901], p. 113004. https://doi.org/10.1103/PhysRevD. 76.113004. https://doi.org/10.1103/PhysRevD.77. 059901. arXiv:0709.4378 [hep-ph]

53. K. M. Graczyk, J. T. Sobczyk, Form factors in the quark resonance model. Phys. Rev. D 77(5) (2008). [Erratum: ibid. 79.7 (2008) p. 079903], p. $\quad 053001$. https://doi.org/10.1103/PhysRevD. 77.053001, https://doi.org/10.1103/PhysRevD.79. 079903. arXiv:0707.3561 [hep-ph]

54. J.A. Nowak, Four momentum transfer discrepancy in the charged current $\pi^{+}$production in the MiniBooNE: Data vs. theory. AIP Conf. Proc. 1189, 243-248 (2009). https://doi.org/10.1063/1.3274164. arXiv:0909.3659 [hep-ph]

55. K.S. Kuzmin, V.V. Lyubushkin, V.A. Naumov, Axial masses in quasielastic neutrino scattering and singlepion neutrinoproduction on nucleons and nuclei. Acta Phys. Polon. B 37(8), 2337-2348 (2006). arXiv:hep-ph/0606184

56. M. S. Alam et al. GENIE Production Release 2.10.0. arXiv:1512.06882 [hep-ph]

57. M. Kabirnezhad, Single pion production in neutrinonucleon interactions. Phys. Rev. D 97(1), 013002 (2018). https://doi.org/10.1103/PhysRevD.97.013002. arXiv:1711.02403 [hep-ph]

58. M. Kabirnezhad, Improvement of single pion production for T2K experiment simulation tools (update). $\mathrm{PhD}$ thesis. National Center for Nuclear Research, Otwock, Świerk, Poland (2020)

59. M. Kabirnezhad, Single pion production in neutrinonucleon interaction. JPS Conf. Proc. 12, 010043 (2016). https://doi.org/10.7566/JPSCP.12.010043

60. M. Kabirnezhad, Single pion production in neutrino reactions. J. Phys: Conf. Ser. 888(1), 012122 (2017). https://doi.org/10.1088/1742-6596/888/1/012122 
61. D. Rein, Angular distribution in neutrino induced single pion production processes. Z. Phys. C 35(1), 43-64 (1987). https://doi.org/10.1007/BF01561054

62. J. Campbell et al., Study of the reaction $\nu p \rightarrow \mu^{-} p \pi^{+}$. Phys. Rev. Lett. 30(8), 335-339 (1973). https://doi. org/10.1103/PhysRevLett.30.335

63. S.J. Barish et al., Study of neutrino interactions in hydrogen and deuterium. II. Inelastic charged-current reactions. Phys. Rev. D 19(9), 2521-2542 (1979). https://doi.org/10.1103/PhysRevD.19.2521

64. G. M. Radecky et al., Study of single-pion production by weak charged currents in low-energy $\nu d$ interactions. Phys. Rev. D /bf 25(5) (1982). [Erratum: ibid. 26.11 (1982) p. 3297], pp. 1161-1173. https://doi.org/ 10.1103/PhysRevD.25.1161. https://doi.org/10.1103/ PhysRevD.26.3297

65. T. Kitagaki et al., Charged current exclusive pion production in neutrino deuterium interactions. Phys. Rev. D 34(9), 2554-2565 (1986). https://doi.org/10.1103/ PhysRevD.34.2554

66. J. Bell et al., Study of the reaction $\nu p \rightarrow \mu^{-} \Delta^{++}$ at high energies and comparisons with theory. Phys. Rev. Lett. 41(15), 1012-1015 (1978). https://doi.org/ 10.1103/PhysRevLett.41.1012

67. J. Bell et al., Cross-section measurements for the reactions $\nu p \rightarrow \mu^{-} p \pi^{+}$and $\nu p \rightarrow \mu^{-} p K^{+}$at high energies. Phys. Rev. Lett. 41(15), 1008-1011 (1978). https:// doi.org/10.1103/PhysRevLett.41.1008

68. D. Allasia et al., Single pion production in charged current $\bar{\nu} D$ interactions at high energies. Z. Phys. C 20(2), 95-100 (1983). https://doi.org/10.1007/BF01573212

69. S. J. M. Barlag, Quasielastic interactions and one pion production by neutrinos and antineutrinos on a deuterium target. PhD thesis, Amsterdam (1984)

70. P.M. Allen et al., A study of single-meson production in neutrino and antineutrino charged-current interactions on protons. Nucl. Phys. B 264(2-3), 221-242 (1986). https://doi.org/10.1016/0550-3213(86)90480-3

71. D. Allasia et al., Investigation of exclusive channels in $\nu / \bar{\nu}$-deuteron charged current interactions. Nucl. Phys. B 343(2), 285-309 (1990). https://doi.org/10. 1016/0550-3213(90)90472-P

72. P. Rodrigues, C. Wilkinson, K. McFarland, Constraining the GENIE model of neutrino-induced single pion production using reanalyzed bubble chamber data. Eur. Phys. J. C 76(8), 474 (2016). https://doi.org/10. 1140/epjc/s10052-016-4314-3. arXiv:1601.01888 [hep$\mathrm{ex}]$

73. E. A. Hawker, Single pion production in low energy neutrino-carbon. Proceedings of the 2nd International Workshop on Neutrino-Nucleus Interactions in the Few $\mathrm{GeV}$ Region (NuInt 2002), Irvine, California, USA, December 12-15, 2002. (2003)

74. E. Wang et al., Photon emission in neutral current interactions at the $\mathrm{T} 2 \mathrm{~K}$ experiment. Phys. Rev. D 92(5), 053005 (2015). https://doi.org/10.1103/ PhysRevD.92.053005. arXiv:1507.02446 [hep-ph]

75. K. Abe et al., Measurement of neutrino and antineutrino oscillations by the $\mathrm{T} 2 \mathrm{~K}$ experiment including a new additional sample of $\nu_{e}$ interactions at the far detector. Phys. Rev. D 96(9) (2017). [Erratum: ibid. 98.1 (2018) p. 019902], p. 092006. https://doi.
org/10.1103/PhysRevD.96.092006. https://doi.org/10. 1103/PhysRevD.98.019902. arXiv:1707.01048 [hep-ex]

76. P. AN Machado, O. Palamara, D.W. Schmitz, The short-baseline neutrino program at Fermilab. Ann. Rev. Nucl. Part. Sci. 69, 363-387 (2019). https://doi.org/10.1146/annurev-nucl-101917-020949. arXiv:1903.04608 [hep-ex]

77. E. Wang, L. Alvarez-Ruso, J.M. Nieves, Photon emission in neutral current interactions at intermediate energies. Phys. Rev. C 89(1), 015503 (2014). https://doi.org/10.1103/PhysRevC.89.015503. arXiv:1311.2151 [nucl-th]

78. E. Saúl Sala, Open problems in the Physics of Neutrino Interactions with Nucleons and Nuclei. PhD thesis. Universidad de Valencia (2021). https://ific.uv.es/ nucth/Thesis_Saul_Sala.pdf

79. H. De Vries, C.W. De Jager, C.P. De Vries, Nuclear charge and magnetization density distribution parameters from elastic electron scattering. Atom. Data Nucl. Data Tabl. 36(3), 495-536 (1987). https://doi.org/10. 1016/0092-640X(87)90013-1

80. J. Cugnon et al., Processes involving few degrees of freedom in the frame of intranuclear cascade approaches. Eur. Phys. J. Plus 131(5), 169 (2016). https://doi.org/10.1140/epjp/i2016-16169-4

81. S. Dytman et al., Comparison of validation methods of simulations for final state interactions in hadron production experiments. arXiv:2103.07535 [hep-ph]

82. L.L. Salcedo et al., Computer simulation of inclusive pion nuclear reactions. Nucl. Phys. A 484(3-4), 557-592 (1988). https://doi.org/10.1016/ 0375-9474(88)90310-7

83. V.R. Pandharipande, S.C. Pieper, Nuclear transparency to intermediateenergy nucleons from (e, $\left.e^{\prime} p\right)$ reactions. Phys. Rev. C 45(2), 791-798 (1992). https:// doi.org/10.1103/PhysRevC.45.791

84. E.S. Pinzon Guerra et al., Using world charged $\pi^{ \pm}$-nucleus scattering data to constrain an intranuclear cascade model. Phys. Rev. D 99(5), 052007 (2019). https://doi.org/10.1103/PhysRevD.99.052007. arXiv:1812.06912 [hep-ex]

85. M.G. Aartsen et al., First observation of PeV-energy neutrinos with IceCube. Phys. Rev. Lett. 111(2), 021103 (2013). https://doi.org/10.1103/PhysRevLett. 111.021103. arXiv:1304.5356 [astro-ph.HE]

86. M.G. Aartsen et al., Evidence for high-energy extraterrestrial neutrinos at the IceCube Detector. Science 342(6161), 1242856 (2013). https://doi.org/10.1126/ science.1242856. arXiv:1311.5238 [astro-ph.HE]

87. M.G. Aartsen et al., Observation of high-energy astrophysical neutrinos in three years of IceCube Data. Phys. Rev. Lett. 113(10), 101101 (2014). https://doi.org/10.1103/PhysRevLett.113.101101. arXiv:1405.5303 [astro-ph.HE]

88. H. Abreu et al., Technical Proposal: FASER $\nu$. arXiv:2001.03073 [physics.ins-det]

89. S. Alekhin et al., A facility to search for hidden particles at the CERN SPS: the SHiP physics case. Rept. Progr. Phys. 79(12), 124201 (2016). https://doi.org/ 10.1088/0034-4885/79/12/124201. arXiv:1504.04855 [hep-ph]

90. A. Garcia et al., Complete predictions for highenergy neutrino propagation in matter. JCAP 2020, 
025 (2009). https://doi.org/10.1088/1475-7516/2020/ 09/025. arXiv:2004.04756 [hep-ph]

91. V. Bertone, S. Carrazza, J. Rojo, APFEL: a PDF evolution library with QED corrections. Comput. Phys. Commun. 185(6), 1647-1668 (2014). https://doi.org/ 10.1016/j.cpc.2014.03.007. arXiv:1310.1394 [hep-ph]

92. V. Bertone, R. Gauld, J. Rojo, Neutrino telescopes as QCD microscopes. JHEP 01, 217 (2019). https:// doi.org/10.1007/JHEP01(2019)217. arXiv:1808.02034 [hep-ph]

93. R.D. Ball et al., Parton distributions with small$\mathrm{x}$ resummation: evidence for BFKL dynamics in HERA data. Eur. Phys. J. C 78(4), 321 (2018). https://doi.org/10.1140/epjc/s10052-018-5774-4. arXiv:1710.05935 [hep-ph]

94. R. Aaij et al., Prompt charm production in $p p$ collisions at $\sqrt{s}=7 \mathrm{TeV}$. Nucl. Phys. B 871(1), 120 (2013). https://doi.org/10.1016/j.nuclphysb.2013. 02.010. arXiv:1302.2864 [hep-ex]

95. R. Aaij et al., Measurements of prompt charm production cross-sections in $p p$ collisions at $\sqrt{s}=13$ TeV. JHEP 03 (2016). [Erratum: ibid. 09 (2016) p. 013, ibid. 05 (2017) p. 074], p. 159. https://doi. org/10.1007/JHEP03(2016)159, https://doi.org/10. 1007/JHEP09(2016)013, https://doi.org/10.1007/ JHEP05(2017)074. arXiv:1510.01707 [hep-ex]

96. R. Aaij et al., Measurements of prompt charm production cross-sections in $p p$ collisions at $\sqrt{s}=5$ TeV. JHEP 06, 147 (2017). https://doi.org/10.1007/ JHEP06(2017)147. arXiv:1610.02230 [hep-ex]

97. S. Forte et al., Heavy quarks in deep-inelastic scattering. Nucl. Phys. B 834(1-2), 116-162 (2010). https://doi.org/10.1016/j.nuclphysb.2010.03.014. arXiv:1001.2312 [hep-ph]

98. A. Cooper-Sarkar, P. Mertsch, S. Sarkar, The high energy neutrino cross-section in the Standard Model and its uncertainty. JHEP 08, 042 (2011). https://doi. org/10.1007/JHEP08(2011)042. arXiv:1106.3723 [hep$\mathrm{ph}]$

99. G. Ingelman, A. Edin, J. Rathsman, LEPTO 6.5: A Monte Carlo generator for deep inelastic lepton-nucleon scattering. Comput. Phys. Commun. 101(1-2), 108-134 (1997). https://doi.org/10.1016/ S0010-4655(96)00157-9. arXiv:hep-ph/9605286

100. M.M. Tzanov et al., Precise measurement of neutrino and anti-neutrino differential cross sections. Phys. Rev. D 74(1), 012008 (2006). https://doi.org/10.1103/ PhysRevD.74.012008. arXiv:hep-ex/0509010

101. R. U. Abbasi et al., Measurement of the high-energy all-flavor neutrino-nucleon cross section with IceCube. Phys. Rev. D 104, 2 (2021). arXiv:2011.03560 [hep-ex]

102. D.Z. Freedman, Coherent neutrino nucleus scattering as a probe of the weak neutral current. Phys. Rev. D 9(5), 1389-1392 (1974). https://doi.org/10.1103/ PhysRevD.9.1389
103. D. Akimov et al., Observation of coherent elastic neutrino-nucleus scattering. Science 357(6356), 11231126 (2017). https://doi.org/10.1126/science.aao0990. arXiv:1708.01294 [nucl-ex]

104. D. Akimov et al., First measurement of coherent elastic neutrino-nucleus scattering on Argon. Phys. Rev. Lett. 126(1), 012002 (2021). https://doi.org/10.1103/ PhysRevLett.126.012002. arXiv:2003.10630 [nucl-ex]

105. E. Ciuffoli et al., Extracting nuclear form factors with coherent neutrino scattering. Phys. Rev. D 97(11), 113003 (2018). https://doi.org/10.1103/PhysRevD.97. 113003. arXiv:1801.02166 [physics.ins-det]

106. N. Van Dessel et al., Nuclear structure physics in coherent elastic neutrino-nucleus scattering. arXiv:2007.03658 [nucl-th]

107. J. Billard, J. Johnston, B.J. Kavanagh, Prospects for exploring new physics in coherent elastic neutrino-nucleus scattering. JCAP 2018, 016 (1811). https://doi.org/10.1088/1475-7516/2018/11/ 016. arXiv:1805.01798 [hep-ph]

108. M. Bowen, P. Huber, Reactor neutrino applications and coherent elastic neutrino nucleus scattering. Phys. Rev. D 102(5), 053008 (2020). https://doi.org/10.1103/ PhysRevD.102.053008. arXiv:2005.10907 [physics.insdet]

109. S. Gardiner, Simulating low-energy neutrino interactions with MARLEY. arXiv:2101.11867 [nucl-th]

110. K. Patton et al., Neutrino-nucleus coherent scattering as a probe of neutron density distributions. Phys. Rev. C 86(2), 024612 (2012). https://doi.org/10.1103/ PhysRevC.86.024612. arXiv:1207.0693 [nucl-th]

111. J. Tena-Vidal et al., AGKY Hadronization Model Tuning in GENIE v3. arXiv:2106.05884 [hep-ph]

112. P. V. Abratenko et al., Measurement of differential cross sections for $\nu_{\mu}$-Ar charged-current interactions with protons and no pions in the final state with the MicroBooNE detector. Phys. Rev. D 102.11 (2020), p. 112013. https://doi.org/10.1103/PhysRevD. 102.112013. arXiv:2010.02390 [hep-ex]. 30 Will be inserted by the editor

113. T.T. Le et al., Measurement of $\bar{\nu}_{\mu}$ charged-current single $\pi^{-}$production on hydrocarbon in the few$\mathrm{GeV}$ region using MINER $\nu \mathrm{A}$. Phys. Rev. D 100(5), 052008 (2019). https://doi.org/10.1103/PhysRevD. 100.052008. arXiv:1906.08300 [hep-ex]

114. J. Berger, A module for boosted dark matter event generation in GENIE. arXiv:1812.05616 [hep-ph]

115. E. Bertuzzo et al., Dark Neutrino Portal to explain MiniBooNE excess. Phys. Rev. Lett. 121(24), 241801 (2018). https://doi.org/10.1103/PhysRevLett. 121.241801. arXiv:1807.09877 [hep-ph]

116. M. Roda, I. de Icaza. Dark neutrino kinematics. GENIE docDB \#206. https://genie-docdb.pp.rl.ac. uk/DocDB/0002/000206/002/Dark_Neutrino.pdf 